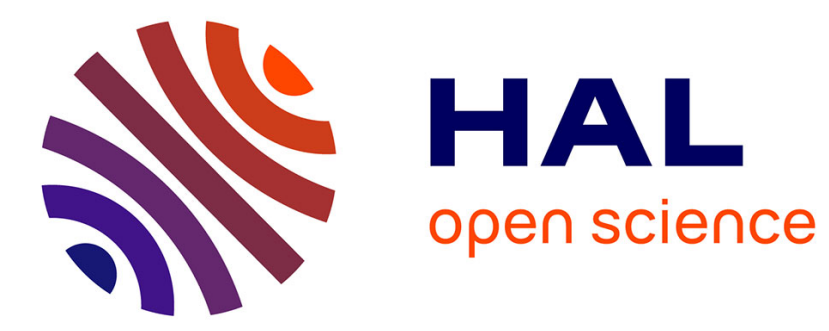

\title{
De l'origine du cobalt : du verre à la céramique
}

Bernard Gratuze, Isabelle Soulier, Maryse Blet, Lucy Vallauri

\section{To cite this version:}

Bernard Gratuze, Isabelle Soulier, Maryse Blet, Lucy Vallauri. De l'origine du cobalt: du verre à la céramique. Archeosciences, revue d'Archéométrie, 1996, 20 (1), pp.77-94. 10.3406/arsci.1996.939. hal-01829843

\section{HAL Id: hal-01829843 \\ https://hal.science/hal-01829843}

Submitted on 5 Jul 2018

HAL is a multi-disciplinary open access archive for the deposit and dissemination of scientific research documents, whether they are published or not. The documents may come from teaching and research institutions in France or abroad, or from public or private research centers.
L'archive ouverte pluridisciplinaire HAL, est destinée au dépôt et à la diffusion de documents scientifiques de niveau recherche, publiés ou non, émanant des établissements d'enseignement et de recherche français ou étrangers, des laboratoires publics ou privés. 


\title{
DE L'ORIGINE DU COBALT: DU VERRE Â LA CÉRAMIQUE
}

\author{
Bernard GRATUZE*, Isabelle SOULIER*, Maryse BLET*, Lucy VALLAURI**
}

\begin{abstract}
Résumé: Dans un article antérieur, nous présentions un modèle d'approvisionnement en cobalt pour l'industrie verrière en France, pour la période s'étalant de l'Antiquité à nos jours. Nous avons récemment étendu cette étude aux glaçures de céramiques. Les premiers résultats que nous présentons ici concement un échantillonnage très varié tant du point de vue géographique que chronologique. Ces résultats confirment et affinent ceux obtenus sur le verre pour la période postérieure au XIIIe siècle. Ils montrent, de plus, que les résultats obtenus sur les verres français peuvent être généralisés à la partie occidentale du Bassin Méditerranéen. Pour la partie orientale de ce bassin, ils ouvrent de nouvelles perspectives de recherche.
\end{abstract}

\begin{abstract}
In a preceding paper dealing with the sourcing of cobalt, we were proposing a trade model for the french glass industry. Since we have extended our study to ceramic glaze. The result we present here are devoted to a selection of sample of broad geographical and chronological origins. These first results confirm those obtained for glass and allow us to spread our model to the whole occidental part of the mediterranean basin. For the oriental part of the Ancient World, they allow some interesting new research projects.
\end{abstract}

Mots-clés: Glaçure, cobalt, bleu, céramique, provenance, analyse, ICP-MS, ablation laser.

Key-words: Glaze, pottery, cobalt, blue, sourcing, analysis, ICP-MS, laser ablation.

\section{INTRODUCTION}

En 1992, nous présentions, dans cette même revue, les premiers résultats d'une étude originale sur la provenance des colorants bleus au cobalt utilisés par les verriers au cours du temps (Gratuze et al., 1992). Depuis lors, plus de 400 objets en verre de périodes et d'origines variées ont été analysés. Ce travail nous a permis de mettre en évidence l'utilisation d'au moins quatre grands types de colorants à base de cobalt, correspondant à différents gisements, sur une période s'étalant de l'Antiquité jusqu'au dix-huitième siècle (Soulier et al., 1996). Ce travail important réalisé sur le verre soulève cependant plusieurs questions sur l'utilisation du cobalt en général. En effet, si le cobalt (sous les noms de safre ou de smalt) a été utilisé pour colorer le verre, il a aussi été employé pour la fabrication des glaçures de céramiques, des émaux et d'une façon plus restreinte des pigments de peintures à l'huile. Il est légitime de se demander si l'étude de ces matériaux aboutirait à des résultats identiques à ceux obtenus pour le verre.

En 1995, l'acquisition par notre laboratoire d'un ICP-MS couplé à une ablation laser nous a ouvert de nouveaux champs d'investigation. En effet, la possibilité d'analyser les constituants majeurs, mineurs et traces d'un objet à partir du prélèvement effectué par le laser (avec un cratère dont le diamètre est compris entre 20 et $100 \mathrm{~mm}$ ) nous a conduits à élargir cette étude aux glaçures de céramiques.

Ce choix nous semble particulièrement judicieux. En effet, par rapport au verre, les céramiques présentent plusieurs avantages:

- les datations et leurs régions de provenance sont établies de façon beaucoup plus précise que dans le cas des verres,

- les ateliers de fabrication sont mieux répertoriés et étudiés, grâce à la caractérisation des argiles,

- contrairement aux verres, il ne peut pas y avoir de réutilisation par refonte,

- le matériel disponible est beaucoup plus abondant, et il existe de nombreuses collections de références de tessons.

Les objectifs de ce programme sont dans un premier temps:

- d'élargir le champ d'investigation géographique de nos précédents travaux,

- d'affiner la chronologie mise en place.

A plus longue échéance, nous souhaitons:

- vérifier si les résultats obtenus sur les glaçures par cette technique sont aptes à résoudre les problèmes d'identification de provenance lorsque l'analyse des pâtes

* Centre de Recherche Ernest Babelon, C.N.R.S., F-45071 ORLE்ANS Cedex 2.

** Laboratoire d'Archéologie Médièvale Méditerranéenne, C.N.R.S., Université de Provence, F-13621 ALX-EN-PROVENCE Cedex 1. 
céramiques échoue,

- élargir les problèmes d'origines des constituants entrant dans la fabricatioñ des glaçures à d'autres éléments comme l'étain ou le plomb (dans certains cas favorables, l'analyse des isotopes du plomb peut être envisagée par cette technique).

Nous présentons ici les premiers résultats de ce travail qui concernent un échantillonnage varié de céramiques à glaçure et/ou à motif bleu.

\section{CHOIXET DESCRIPTION DESÉCHANTTLLONS}

Notre choix peut, à première vue, paraitre assez disparate. Il a cependant été dicté par l'objectif de cette recherche qui consiste à vérifier si les observations effectuées à partir des verres sont confirmées par les résultats obtenus sur les glaçures bleues. Il présente donc par conséquent une grande variabilité tant géographique que chronologique.

Pour cette première étude, nous avons sélectionné une cinquantaine d'échantillons datés du XIIème au XVIIIème siècle provenant d'ateliers français (Région Centre, Région lyonnaise et Provence) et de l'ensemble du bassin méditerranéen (Maghreb, Espagne, Italie, Syrie et Egypte) mais aussi d'Ouzbékistan (Samarkand). Les échantillons provenant du Maghreb, du Proche-Orient, d'Italie et d'Espagne sont pour la plupart des céramiques d'importation trouvées en Provence (Marseille, Hyères, Avignon, Aix-en-Provence, Rougiers...) mais dont l'origine est confirmée par les analyses géochimiques du Laboratoire de Céramologie de Lyon, C.N.R.S. (Démians d'Archimbaud et Picon, 1980). A l'exception des tessons glaçurés de la région Centre prêtés par P. Bon (Bon, 1992) et des deux fragments de sculpture de Della Robbia prêtés par J.R. Gaborit, l'ensemble des échantillons analysés provient du fonds de documentation réuni par le Laboratoire d'Archéologie Médiévale Méditerranéenne.

\section{Les échantillons proviennent:}

- du château de Mehun-sur-Yèvre; l'ensemble des céramiques architecturales trouvées lors des fouilles de ce château a été étudié par P. Bon (Bon, 1992). Les carreaux ont peut-être été fabriqués à Bourges vers la fin du XIVème siècle. Une documentation complète a été réunie par $\mathrm{P}$. Bon sur les ateliers de fabrication de ces carreaux pour le Duc Jean de Berry. Dans le cas de l'un de ces ateliers, celui de Poitiers, il y est en particulier fait mention de l'achat pour 16 sols "A Perrot Mehé pour une livre de saffre neccessaire pour poindre les quarreaux" (sic) le 4 juin 1386. Le même artisan (Jehan de Valence, dit le Sarrazin) ayant probablement mis en place les deux ateliers, on peut supposer que le safre a aussi été utilisé à Bourges pour colorer les carreaux en bleu.

Six échantillons provenant des fouilles du château de Mehun-sur-Yèvre ont été analysés:

- MY1, carreau bleu à pâte blanchâtre présentant un retour des coulures par le dessous (parcelle 87, devant les caves),

- MY2, carreau uni bleu à pâte blanchâtre-rosée (parcelle 87),

- MY3, carreau turquoise sur pâte rose (douves),

- MY 4, carreau, élément de fleurs de lys blanches sur fond bleu et engrelure brun-rouge, pâte blanchâtre (douves),

- MY5, carreau, élément de la queue d'un oiseau, contour bleu sur fond blanc, pâte blanche (pont d'entrée du site),

- MY6, fragment de carreau lobé bleu sur support blanc, pâte rouge (pont d'entrée du site).

- du Louvre, sculptures d'Andréa Della Robbia (1435-1525)

Deux échantillons témoins d'une reproduction de la "bottega" des Della Robbia datables du dernier quart du XVème siècle ou du début du siècle suivant:

-DB1, fragment de terre cuite émaillée (bleu gris) trouvé dans le fourrage en plâtre du panneau avec deux têtes de chérubins (numéro d'inventaire: Campana 72),

-DB2, fragment de terre cuite émaillée (bleu vif) trouvé dans le fourrage en plâtre du panneau de l'ange volant (numéro d'inventaire: Campana 65),

\section{- du Laboratoire d'Archéologie Médiévale Méditerranéenne.}

[Les échantillons sont regroupés ici par origine géographique des productions]

* Provence, trois échantillons datés de la fin XVIIèmeXVIIIème siècle:

- FP1, fragment d'encrier peint en bleu, faïence de Moustiers, Roquefeuille, Pourrières, Var, réf. 11330 (Foy et al., 1995),

- FP2, bassin à décor bleu, faïence de Varages (Var), Roquefeuille, Pourrières, Var, réf. 12481 (Foy et al., 1986),

-FP3, fond d'assiette à décor bleu, faïence d'Avignon, dépotoir d'atelier rue des Trois Colombes, (Carru et al., 1995).

* Lyon, trois échantillons du XVIème siècle:

-FL1, fragment avec décor de feuilles de vigne bleues sur fond blanc, première moitié du XVIème s., Place de la République, dépotoir d'atelier (Amouric et al., 1997 à paraître),

-FL2, fragment de ronde bosse, glaçure monochrome bleue, seconde moitié du XVIème s., Place des Terreaux (Amouric et al., 1997 à paraître),

-FL3, fragment de vase à décor polychrome, bleu et vert sur fond blanc, seconde moitié du XVlème s., Place des Terreaux (Amouric et al., 1997 à paraître).

* Italie du Sud (Brindisi?), quatre échantillons datés de la seconde moitié du XIIIème siècle:

- IB1 et IB2, fragment de coupe à décor bleu et brun et fragment de coupe à marli, décor bleu et brun, Marseille (Cathédrale), réf. (687 26 124) 1079 et 1075, (Moliner, 1990),

- IB3, fragment de coupe à marli, décor bleu et brun, Marseille (Sainte-Barbe), réf. (384) 19654 (Vallauri, 1997à paraître),

- IB4, fragment de marli, décor bleu et brun, Rougiers, Var, réf. 4120 (Démians D’Archimbaud, 1981).

* Italie, Ligurie (Savone), deux échantillons datés du XVlème siècle:

- ISI et IS2, fragments de coupe à décor bleu peints respectivement sur fond bleu clair et sur fond blanc sur les deux faces, Marseille, réf. 10283-3 et 10283-4.

* Italie, Florence (Monteluppo), trois échantillons datés des XIVème et XVIème siècles:

- IF1, fragment de col de cruche à décor bleu et brun, XIVème s., Marseille (Jules Verne), réf. JV5 29620335 (Hesnard et al., 1993),

- IF2 et IF3, fragment de coupe à décor bleu, jaune et orange et fragment de cruche à décor bleu, violet et orange 
sur les deux faces, XVIème s., Marseille.

* Espagne du Sud (ivíaiāgà), deux échantillons datés de la fin du XIIİme, début du XIVème siècle:

-EM1, fragment de coupe à décor bleu, Hyères Olbia, réf. (810), 5286, (Démians d'Ârchimbaud et Lemoine, 1980),

- EM2, fragment de vase à anse horizontale, décor bleu, Marseille (Saint-Victor), réf. 6999, (Démians d'Archimbaud et Lemoine, 1980).

* Espagne (Valence), cinq échantillons du groupe C (début du XIVème siècle) et quatre échantillons classiques (seconde moitié XIVème et début XVème siècle).

Groupe C:

$-E V 1, E V 2, E V 3$ et EV4, fragments de coupes à décor bleu, Rougiers, réf. 4063 (bleu), 4064 (bleu et lustre), 4065 (bleu), 4106 (bleu et lustre), (Démians d'Archimbaud et Lemoine, 1980),

-EV5, fond de coupe creuse à décor bleu, Hyères Olbia, réf. 5273, lustre disparu? (Démians d'Archimbaud, 1981),

Classique:

- EV6, fragments de bol à décor de croisillons bleus, seconde moitié XIVème s., Rougiers, réf. 4008, (Démians d'Archimbaud, 1981),

-EV7, EV8 et EV9, fragments de bols, respectivement palmettes bleues (bleu seul), Ave Maria bleu et fleurs bleues (lustre disparu?), Marseille (Sainte-Barbe), fin XIVème-XVème s., réf. (137) 20521, (105) 20522 et (120) 20523, (Vallauri, 1997 à paraître).

* Espagne (Barcelone), cinq échantillons:

-EB1, fond de bol à décor bleu, XVème s., Marseille, réf. $10283-6$,

-EB2, rebord de bol à décor bleu, XVème s., Barcelone, réf 6574

- EB 3 et EB4, carreaux à décors respectifs bleu et jaune d'une part et bleu d'autre part, XVIIème s., Aix-enProvence (Archevêché), réf. 20524 et 20525 (Fixot et al., 1986),

-EB5, style Barcelone XVème s., origine indéterminée (locale, Espagne ou Italie), carreau à décor bleu, Avignon (Hôtel de Brion), réf. 5385, (Démians d'Archimbaud et al., 1980).

* Maghreb (Tunisie), trois échantillons datés des XIIÌme-XIVème siècles:

- MT1 et MT2, fragments de coupe à décor bleu et brun, Marseille (Sainte-Barbe), réf. (209)20526 et (384) 20527 (Vallauri, 1997, à paraître),

-MT3, fragment de coupe à décor bleu et brun, Hyères Olbia, réf. (162) 5144 (Parent, 1991),

* Tessons orientaux trouvés en Provence, trois échantillons:

- OR1, fragment de coupe à marli, glaçure bleue sur les deux faces, fin XIlèmes., Marseille(César), réf(3114) 20520 (Abel, 1996),

- OR2, fragment de col de vase à décor bleu, XIVème s., Marseille (Sainte-Barbe), réf. (107) 19884, (Vallauri, 1995; Thiriot, 1995),

- OR3, fragment de vase à décor bleu sur les deux faces, XIVème s., Saint-Martin-de-la-Brasque, réf. 7662, (Thiriot, 1991).

* Ouzbékistan (Samarkand), quatre échantillons:

- SA1, fragment de brique à glaçure bleue, Nécropole
Shah-e-Zende, fin XIVème s., restauration XXe s.?, - SA2, brique d'incrustation à glaçure bleue, Ishrat Khan, milieu XVèmes.,

- SA3, fragment de vase à décor bleu sur émail, Gour Emir, XVIèmes.,

-SA4, fragment de brique à glaçure bleue, Ulugh Beyg, restauration XXèmes. ?

* Syrie (Meskéné), quatre échantillons médiévaux provenant du site:

- SM1, fragment de glaçure bleu turquoise, four de potier, milieu XIIlèmes. (Thiriot, à paraître)

- SM2, tessons à glaçure bleue sur les deux faces,

- SM3, fragment de vase à décor bleu et lustre à l'extérieur,

- SM4, fragment de coupe à décor bleu et lustre sur les deux faces.

* Egypte (Fustat), deux échantillons médiévaux XIème-

XIIème siècle?:

-EG1, fragment de coupe esgrafiée et côtelée, glaçure bleue sur les deux faces, réf. 76030 ,

-EG2, fragment de vase, glaçure bleue à l'extérieur et verte à l'intérieur, réf. 76021 .

Cet échantillonnage apparaît suffisant pour vérifier les hypothèses posées lors de nos travaux sur les verres pour la période allant du XIIème au XVIIIème siècle. La comparaison effectuée avec les échantillons orientaux nous permet d'ouvrir nos investigations à des régions encore non explorées lors de nos précédentes recherches.

\section{MÉTHODED'ANALYSE}

La méthode d'analyse utilisée lors de cette étude est la spectrométrie de masse couplée à un plasma inductifavec prélèvement par ablation laser (LA-ICP-MS pour Laser Ablation Inductively Coupled Plasma Mass Spectrometry). Cette technique est décrite dans un article antérieur (Gratuze et al., 1993), des changements mineurs sont toutefois à signaler par rapport au travail précédent:

- L'appareil utilisé ici est un "VG PlasmaQuad 2" version PQXS couplé depuis le début de l'année 1996 à un système d'ablation laser "UVMicroProbe" (le laser était infra-rouge dans un premier temps).

- Le laser UV est un laser néodyme YAG dont la fréquence est quadruplée, sa longueur d'onde est donc de $266 \mathrm{~nm}$. Il est utilisé en mode Q-switched. Le diamètre des ablations réalisées varie entre 20 et $80 \mathrm{~mm}$ selon l'épaisseur de la glaçure étudiée, la profondeur du cratère varie entre 250 et $300 \mathrm{~mm}$. Les ablations sont effectuées dans une cellule en quartz de $5 \mathrm{~cm}$ de diamètre. Un microscope permet de choisir très précisément la zone de prélèvement.

Les limites de détection varient en fonction de la taille de l'impact. Pour la plupart des éléments traces étudiés lors de ce travail, elles sont de l'ordre de la ppm et sont parfois même inférieures. Pour les analyses quantitatives, la précision des résultats dépend beaucoup du matériau étudié. Dans les matériaux vitreux homogènes, elle varie entre 5 et $10 \%$ relatifs. Dans le cas des glaçures stanniferes, la précision peut être de l'ordre de $20 \%$ ou plus, du fait de la grande hétérogénéité de ce matériau à l'échelle du prélèvement. Lors des calculs, les points présentant une teneur excessive en étain (ablation d'un gros précipité de $\mathrm{SnO} 2)$ ont été éliminés.

Deux types d'analyses ont été réalisés:

- des analyses quantitatives qui donnent la composition 
moyenne de la glaçure lorsque celle-ci est homogène, ou un aperçu de celle-ci lorsque le matériel est trop hétérogène. Les éléments recherchés sonit principalement: le sodium, le potassium, le calcium, l'aluminium, le silicium, le plomb, l'étain, le fer, le manganèse, le cobalt, le nickel, l'arsenic, le zinc, l'indium, le bismuth, le cuivre, l'antimoine, le phosphore, le chlore, le baryum, le chrome, le tungstène, le molybdène et l'uranium. Ces trois demiers éléments sont, d'après les données géochimiques, susceptibles de caractériser les minerais du district de Schneeberg: on note la présence de minerais de tungstène, molybdène et uranium au sein des formations cobaltiferes (De Launay, 1913),

- des profils ou des répartitions de concentration qui permettent de confirmer les corrélations mises en évidence par les analyses quantitatives. Deux exemples seront donnés, l'un pour une glaçure de Lyon (FL3) et l'autre pour une glaçure du Proche-Orient (OR3).

Pour les analyses quantitatives, l'étalonnage de l'appareil a été effectué en utilisant les verres étalons NIST610 et 612 (matériel de référence du National Institute of Standards and Technology) et des verres opacifiés à l'étain (verre relativement homogène, l'étain y étant dispersé très finement) provenant de la verrerie médiévale de $\mathrm{La}$ Seube et analysés par activation avec les neutrons rapides de cyclotron par notre laboratoire (Gratuze, Barrandon et al. 1992; Soulier, 1997).

\section{PRÉPARATION DES ÉCHANTILLONS}

L'analyse, par cette technique, d'échantillons homogènes et massifs pose relativement peu de problèmes. Elle permet, dans la plupart des cas, une analyse quasiment non destructive sans aucune préparation de l'échantillon (dans la mesure où celui-ci tient à l'intérieur de la cellule d'ablation). Il n'en est pas de même dans le cas de l'analyse de glaçures qui sont souvent relativement fines (épaisseur parfois inférieure à la profondeur des ablations) et parfois multicouches. Pour ces raisons, l'analyse est effectuée sur la tranche de l'objet. Le tesson de céramique est coupé à l'aide d'une scie diamant de façon à obtenir une lame épaisse de l'échantillon (quelques millimètres). La surface obtenue est ensuite polie à l'aide d'un abrasif fin (carbure de silicium 1200 mesh) pour éliminer les éventuelles pollutions et les irrégularités de surface (micro-éclatements) dues au sciage. Ce polissage est très important dans le cas des glaçures multicouches et des glaçures fines. Il permet d'obtenir une précision de repérage plus importante sous microscope et donc une analyse plus fine des structures rencontrées.

Pour les glaçures épaisses, un simple nettoyage de la surface de coupe à l'aide d'un bain à ultrasons s'avère souvent suffisant. L'échantillon est ensuite séché.

\section{RÉSULTATS}

Dans le cas des verres, nous avons mis en évidence l'existence de quatre grands groupes de colorants à base de cobalt (tab. 1).

Le premier, qui regroupe l'ensemble des verres datés de la protohistoire jusqu'au XIIème siècle, se caractérise par l'absence d'éléments traces liés au cobalt. Pour certains verres de ce groupe, une corrélation peut parfois être mise en évidence entre le cobalt et le manganèse. Mais, comme ce dernier peut être aussi ajouté intentionnellement comme décolorant, il est parfois difficile de faire la différence entre le manganèse amené par le cobalt et celui ajouté par le verrier pour neutraliser la coloration due au fer. Dans d'autres verres de ce groupe, on peut noter aussi la présence d'antimoine. Celui-ci provient certainement de la refonte de tesselles de mosaïque - où l'antimoine joue le rôle d'opacifiant -, ou de verres dans lesquels ce dernier était utilisé comme décolorant. Ce premier groupe est cependant caractérisé par la présence systématique de cuivre. Toutefois, l'absence de corrélation entre le cobalt et cet élément nous amène à penser qu'ils sont ajoutés séparément. Nous ne pouvons pas pour l'instant proposer une région d'origine pour le cobalt de ce groupe.

Un second groupe rassemble les verres fabriqués entre le début du XIIIème siècle et la fin du XVème siècle (la majorité des verres de ce groupe étant datée XIIIèmeXIVème siècle), et est caractérisé par la présence de zinc, de plomb et d'indium. Ce cobalt provient probablement des mines de la région de Freiberg -Erzgebirge - exploitées vers 1162-1168 (De Launay, 1913; Bari et Becke, 1985).

Dans le troisième groupe, on retrouve essentiellement des verres fabriqués pendant le dernier quart du XVème siècle et le premier quart du XVIème siècle. Il faut aussi signaler le rattachement à ce groupe de trois verres datés du début du XVème siècle. Les verres de ce groupe se caractérisent par des teneurs très élevées en nickel. Une origine allemande - Erzgebirge - est probable pour ce groupe (Soulier, 1997). On peut en effet supposer que son origine est identique à celle du minerai du groupe suivant, et que seule diffère le mode de préparation du pigment (grillage plus ou moins important).

Le quatrième groupe réunit enfin les verres fabriqués à partir du début du XVIème siècle (il est à noter là aussi la présence dans ce groupe de quelques verres datés de la fin du XVème siècle). De fortes teneurs en arsenic, en nickel et occasionnellement en bismuth caractérisent ce groupe. Le minerai de cobalt de ce groupe provient probablement du district minier allemand de Schneeberg - Erzgebirge(Soulier, 1997).

Les attributions de provenance ont été effectuées à partir de données géochimiques qualitatives (De Launay, 1913)

\begin{tabular}{|c|l|l|l|}
\hline Groupe & $\begin{array}{l}\text { Association } \\
\text { chimique }\end{array}$ & $\begin{array}{l}\text { Extension } \\
\text { chronologique }\end{array}$ & $\begin{array}{l}\text { Origine } \\
\text { supposée }\end{array}$ \\
\hline 1 & $\begin{array}{l}\text { Co, Co-Sb ?, } \\
\text { Co-Cu ?, } \\
\text { Co-Mn? }\end{array}$ & $\begin{array}{l}\text { de la } \\
\text { protohistoire au } \\
\text { XIle s. }\end{array}$ & $\begin{array}{l}\text { inconnue, } \\
\text { locale?, Orient? }\end{array}$ \\
\hline 2 & Co-Zn-Pb-In & XIIe - XVe s. & $\begin{array}{l}\text { Freiberg? } \\
\text { (Erzgebirge) }\end{array}$ \\
\hline 3 & Co-Ni & autour de 1500 & Erzgebirge ? \\
\hline 4 & Co-As-Ni-Bi & XVe-XVIIIe s. & $\begin{array}{l}\text { Schneeberg } \\
\text { (Erzgebirge) }\end{array}$ \\
\hline
\end{tabular}


et de textes anciens (Soulier, 1997). Nous ne disposons pas à ce jour d'analyses quantitatives des minerais anciens exploités dans les Mơnts Muétallifères (Erzgebirge). Seule la présence ou l'absence de certaines paragénèses - du type B.G.P., blende-galène-pyrite - nous a servi à étayer nos hypothèses. Par exemple, l'attribution du groupe 2 au district minier de Freiberg a été établie à partir de trois éléments:

- présence de cobalt au sein de la paragénèse B.G.P.,

- coïncidence entre le début de l'exploitation de ces mines (vers 1162-1168) et la mise en évidence de l'utilisation de cette sorte de cobalt au début du XIIIème s.,

- découverte en 1863 de l'indium au sein des blendes de Freiberg.

Sous le terme de district minier de Freiberg, nous désignons l'ensemble des mines produisant du cobalt au sein de paragénèses B.G.P., exploitées à cette époque dans les Monts Métalliferes.

Il faut aussi noter que les corrélations, mise en évidence par notre étude, entre un élément donné et le cobalt ne sont pas toujours liées à la composition du minerai de cobalt exploité (comme c'est le cas pour As et $\mathrm{Ni}$ ) mais proviennent aussi d'associations minérales au sein des filons (cas par exemple de $\mathrm{Zn}$, In, $\mathrm{Pb}, \mathrm{U}, \mathrm{Bi}, \mathrm{Mo}$ et $\mathrm{W}$ ). Dans ce demier cas, la dispersion des teneurs rencontrées est beaucoup plus importante car elle est liée à la composition locale du filon et au mode de préparation du pigment (extraction, tri et purification du minerai de cobalt). La présence ou l'absence d'un élément sera alors l'un des facteurs d'attribution à un groupe.

L'interprétation des résultats obtenus à partir des glaçures est plus délicate que dans le cas des verres. En effet, les glaçures étudiées se divisent en deux grands types (tab. 2). Le premier est caractérisé par des glaçures à base de verre calco-alcalin, et le second, qui se subdivise en deux sous-types, par les glaçures plombifères et plombostanniferes (Blet et al., à paraître).

Dans notre échantillonnage, les glaçures calco-alcalines se retrouvent essentiellement sur les tessons originaires du Proche-Orient (à l'exception de OR1 qui est de type plombo-stannifere), de Syrie et de Samarkand (SA2 et SA3); les glaçures plombifères ont été rencontrées sur les tessons modernes de Samarkand, sur des tessons égyptiens (EGI) et aussi dans les couches de pigment (cas de la peinture sous émail, cf. ci-dessous). L'ensemble des autres glaçures est de type plombo-stannifère.

Si pour le premier type de glaçures, la matrice est la même que celle des verres calco-alcalins déjà étudiés, il n'en est pas de même pour les autres. Nous devons en effet dans ce cas-là tenir compte des éventuelles impuretés amenées par l'étain (indium, tungstène?) ou le plomb (bismuth, zinc, antimoine?). Seule l'analyse des autres couleurs (blanches et autres) pourra nous permettre d'attribuer de façon certaine une glaçure bleue aux groupes définis lors de notre travail sur le verre. Une étude de l'ensemble des couleurs a donc été effectuée pour les tessons à décor polychrome.

Par ailleurs, les glaçures, contrairement aux verres, sont des matériaux relativement hétérogènes. A l'hétérogénéité due à la présence de précipité d'oxyde d'étain que nous avons déjà signalée, s'ajoute un second type d'hétérogénéité, lié à la mise en place de la glaçure ellemême. En effet, les deux grands modes d'application des glaçures se retrouvent dans les échantillons étudiés:

- trempage dans un bain d'émail pré-coloré, dans ce cas la répartition du colorant est assez homogène, seule l'hétérogénéité liée à l'étain est présente dans le cas d'une glaçure plombo-stannifere (photo 1),

- trempage dans un bain d'émail blanc ou pré-coloré et application de la couleur au pinceau par dessus. La zone riche en colorant se trouve à la surface de l'échantillon et diffuse vers l'intérieur (photo 2). Les problèmes liés à l'hétérogénéité de l'opacifiant sont identiques à ceux rencontrés précédemment.

D'autre part, il semblerait qu'une troisième technique ait été utilisée pour certains échantillons - MY5, MY4, MY6, EM1, EV3, EV6, EV9 et peut-être aussi pour EB5 et IS2 -:

- application d'une couleur sur la pâte céramique "au pinceau" puis trempage dans un bain de glaçure incolore ou opacifiée blanche. La couleur diffuse alors vers la couche d'émail, on a donc une hétérogénéité due au profil de diffusion des éléments colorants au sein de la glaçure. La zone riche en colorant se trouve dans ce cas à l'interface glaçure-pâte (photos 3, 4). Pour l'un de ces échantillons MY6 - il se pourrait même que deux trempages successifs aient été effectués (photo 5). Cette technique est habituellement utilisée pour les glaçures non opacifiées, comme c'est le cas pour la coupe SM4 (couleur bleue peinte sous la glaçure tranparente et lustre métallique).

On peut noter ici que nous rencontrons la technique de peinture sous émail opacifié à la fois pour les carreaux de Mehun-sur-Yèvre et pour les tessons provenant du sud de l'Espagne. Or - est-ce une coïncidence? - l'artisan du Duc de Berry vient du Sud de l'Espagne comme l'indique son nom - Jehan de Valence - et, comme les tessons espagnols, les carreaux de Mehun-sur-Yèvre sont datés du XIVème siècle. On peut donc supposer que cette technique, somme toute moins pratique et performante lorsque l'émail est opacifié, a pu être utilisée par les artisans

\begin{tabular}{|c|c|c|c|c|c|c|c|c|c|}
\hline \multirow{3}{*}{$\begin{array}{l}\text { Type } \\
\text { Sous-type } \\
\text { Composition \% } \\
\end{array}$} & \multirow{2}{*}{\multicolumn{3}{|c|}{$\begin{array}{c}1 \\
\text { Calco alcalin } \\
\end{array}$}} & \multirow{2}{*}{\multicolumn{6}{|c|}{ Plombo-stannifère }} \\
\hline & & & & & & & & & \\
\hline & Moyenne & Min & $\operatorname{Max}$ & Moyenne & Min & Max & Moyenne & Min & Max \\
\hline $\mathrm{SiO}_{2}$ & 67 & 50 & 75 & 42,5 & 25 & 75 & 38 & 15 & 60 \\
\hline $\mathrm{PbO}$ & & & $<2$ & 37 & 6 & 70 & 40 & 20 & 70 \\
\hline $\mathrm{Na} 2 \mathrm{O}$ & 12 & 6 & 13 & 2,8 & 0,5 & 8,3 & 1,5 & 0,5 & 6 \\
\hline $\mathrm{K} 2 \mathrm{O}$ & 3,7 & 1 & 10 & 3,1 & 0,6 & 8 & 3,8 & 0,8 & 8 \\
\hline $\mathrm{CaO}$ & 6,8 & 3 & 10 & 2,7 & 0,7 & 8 & 2,7 & 0,6 & 10 \\
\hline $\mathrm{SnO} 2$ & & & $<0,05$ & & & $<2$ & 9 & 2 & 45 \\
\hline $\mathrm{MgO}$ & 2,4 & 0,5 & 4 & 0,4 & 0,1 & 1 & 0,4 & 0,1 & 1 \\
\hline $\mathrm{Al} 2 \mathrm{O} 3$ & 2,4 & 1,2 & 3,6 & 2,5 & 0,7 & 5 & 2,2 & 0,1 & 5,5 \\
\hline $\mathrm{Fe} 2 \mathrm{O} 3$ & 1,6 & 0,2 & 5 & 2,2 & 0,2 & 4 & 1,4 & 0,2 & 5,4 \\
\hline $\mathrm{Cl}$ & 1,2 & 0,4 & 2,1 & 0,4 & 0,2 & 1,7 & 0,4 & 0,1 & 1,3 \\
\hline
\end{tabular}

Tableau 2: Variations de composition pour les principaux oxydes majeurs et le chlore des diffërents types de glaçures rencontrées au cours de cette étude. 


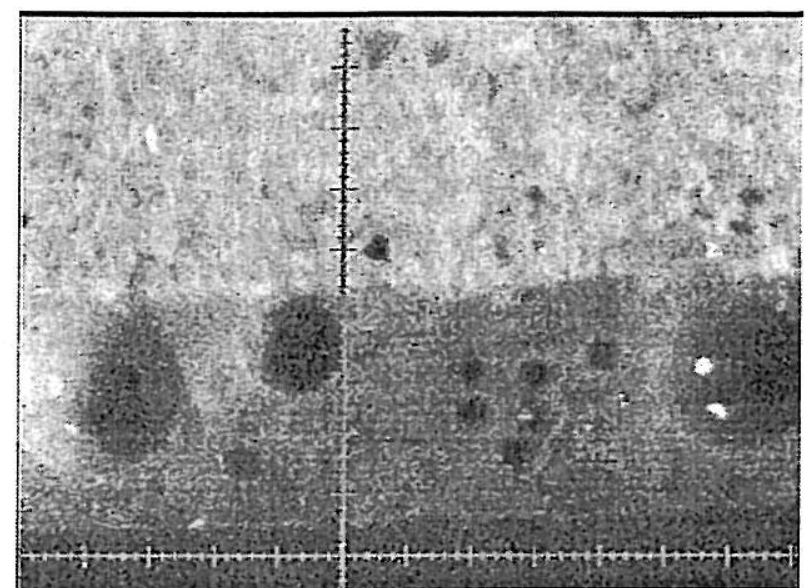

Photo 1 : émaillage par trempage, tesson MY2.

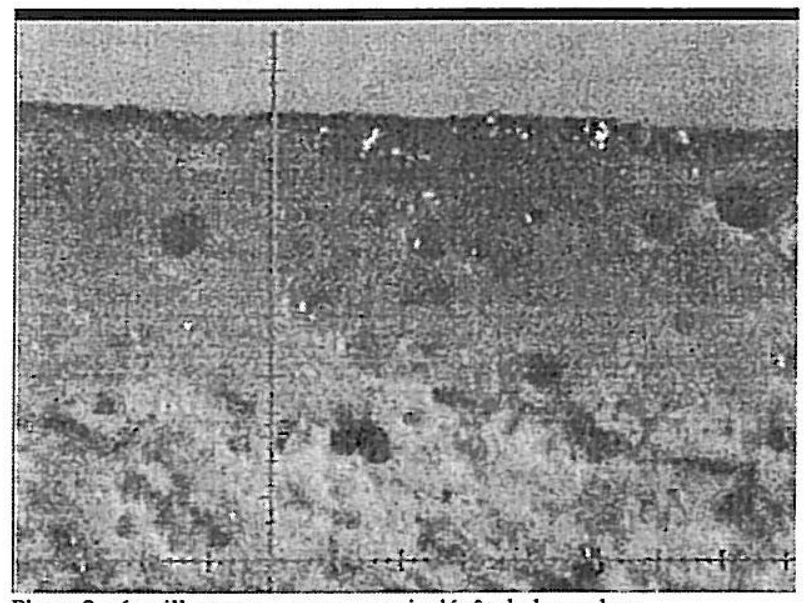

Photo 2 : émaillage par trempage puis dépôt de la couleur au pinceau, tesson FP1.

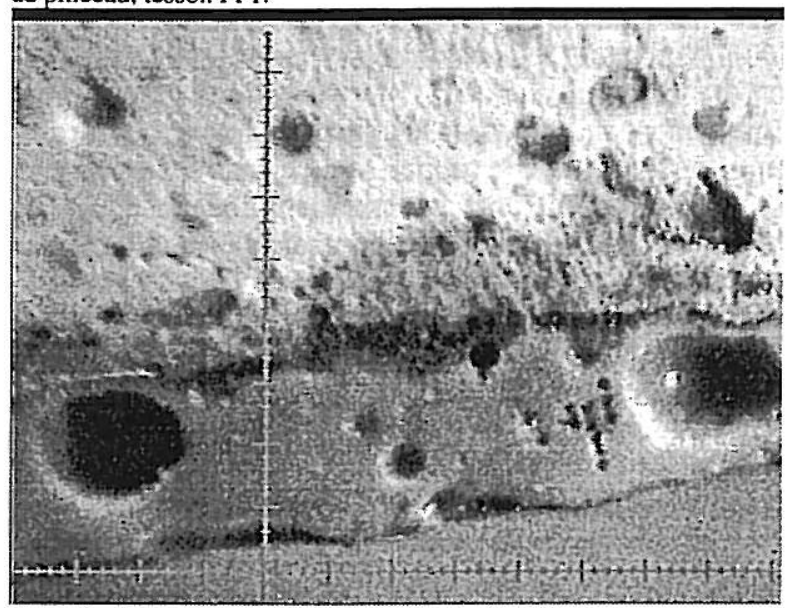

Photo 3 : dépôt au pinceau de la couleur et émaillage par trempage, tesson MY5.

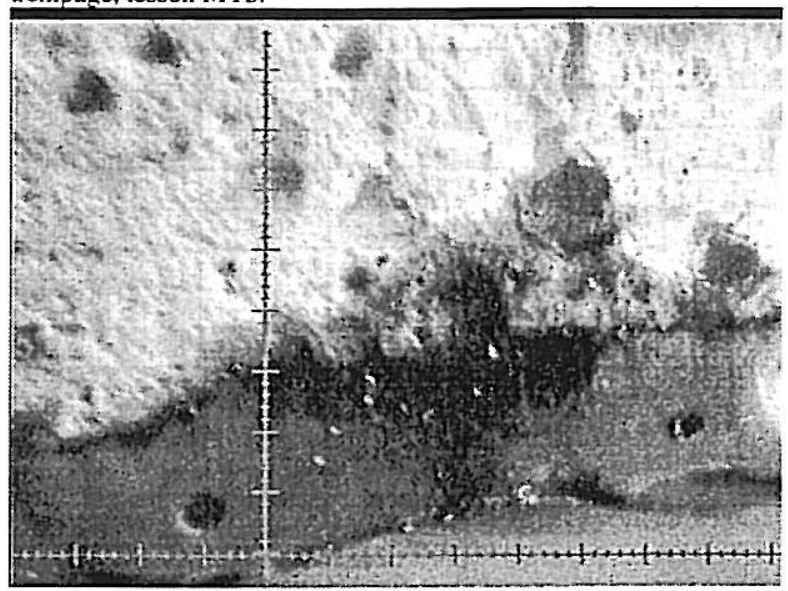

Photo 4 : dépôt au pinceau de la couleur et émaillage par trempage, tesson MY5.

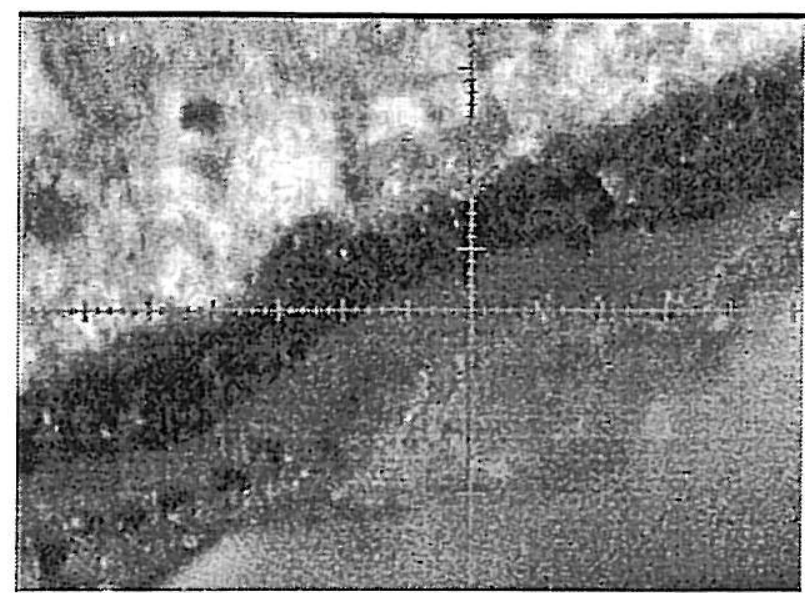

Photo 5 : dépôt de la couleur (pinceau ou trempage) et émaillage par trempage, tesson MY6.

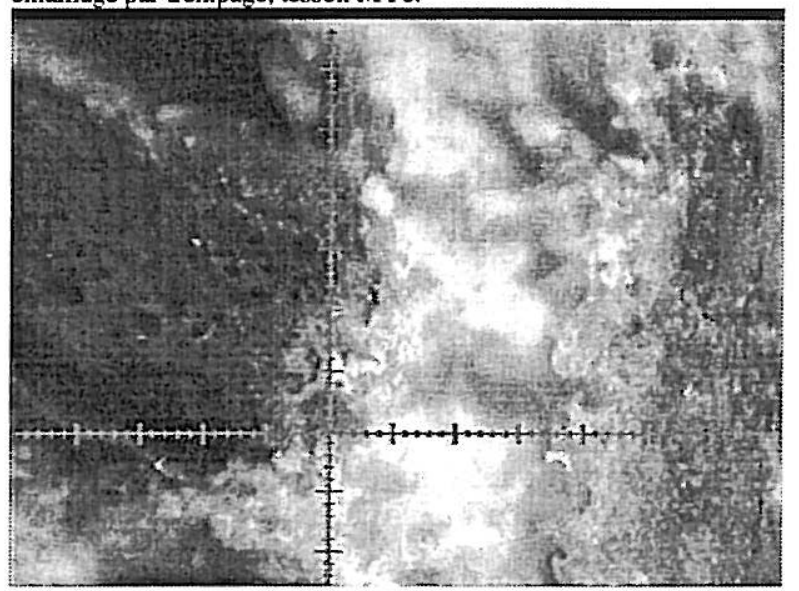

Photo 6: vue d'ensemble, grain de couleur, pâte et glaçure du tesson OR3.

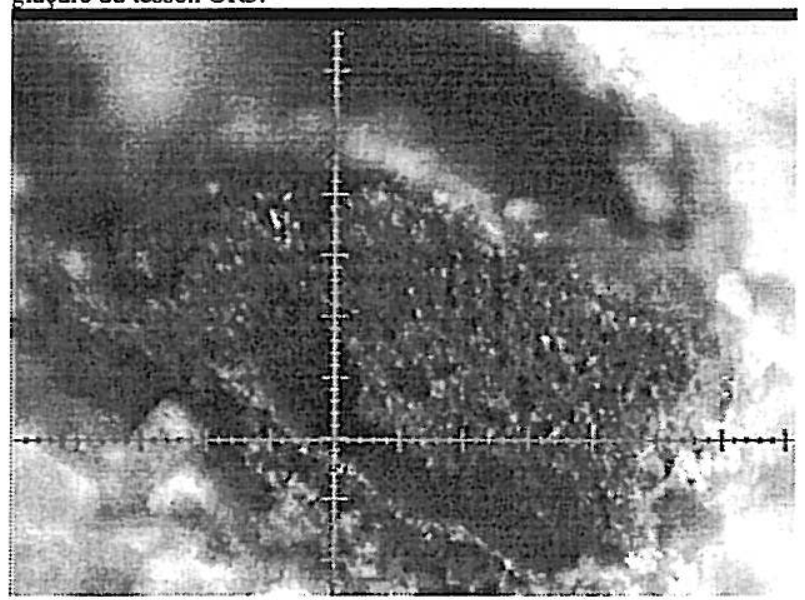

Photo 7: vue du grain de couleur du tesson OR3.

Echelle

Pour les photos $3,4,6,7$, la distance entre deux marques principales est de $110 \quad \mu \mathrm{m}$.

Pour les photos 1,2 et 5 la distance entre deux marques principales est de $45 \mu \mathrm{m}$.

Les cratères d'ablations visibles sur les photos 1, 3, 4 et 7 ont un diamètre compris entre 30 et $40 \mu \quad \mathrm{m}$.

L'absence de zone de diffusion pour le tesson MY6, (photo 5) contrairement au tesson MY5 (photo 2 et 3 ) permet d'envisager l'utilisation d'une technique différente pour la mise en oeuvre de la couleur. 


\begin{tabular}{|c|c|c|c|c|c|c|c|c|c|c|c|c|c|c|c|c|c|}
\hline Référence & \begin{tabular}{|l|} 
Epoque \\
\end{tabular} & Origine & \begin{tabular}{|l|} 
Type et \\
couleur
\end{tabular} & Co & $\mathrm{Zn}$ & In & $\mathrm{Ni}$ & As & $\mathrm{Bi}$ & $\bar{W}$ & Mo & $\bar{U}$ & $\mathrm{Cr}$ & $\mathrm{Fe}$ & $\mathrm{Mn}$ & $\mathrm{Cu}$ & $\mathrm{Sb}$ \\
\hline EM1 & XIU-XIV & Espagne & $\mathrm{g} \mathrm{W}$ & 2 & 94 & 6 & 45 & 34 & 7 & 1 & 0 & 2 & 52 & 11097 & 69 & 98 & 471 \\
\hline EM2 & XIII-XIV & Espagne & $\mathrm{gW}$ & 14 & 250 & 2 & 13 & 11 & 1 & 1 & 1 & 1 & 11 & 4569 & 179 & 290 & 8 \\
\hline EV1 & XIV & Espagne & $\mathrm{gW}$ & 26 & 53 & 11 & 16 & 9 & 12 & 125 & 0 & 2 & 7 & 3751 & 69 & 189 & 34 \\
\hline EV2 & XIV & Espagne & $\mathrm{gW}$ & 65 & 87 & 5 & 1 & 11 & 8 & 59 & 0 & 1 & 3 & 3009 & 74 & 557 & 266 \\
\hline EV6 & XIV & Espagne & $\mathrm{gW}$ & 19 & 173 & 3 & 12 & 22 & 10 & 2 & 1 & 2 & 11 & 5979 & 77 & 1803 & 6 \\
\hline IB1 & XIII & Italie & $\mathrm{g} \mathrm{W}$ & 11 & 685 & 16 & 59 & 12 & 11 & 1 & 1 & 1 & 46 & 6068 & 327 & 131 & 301 \\
\hline $\mathrm{IB} 2$ & XIII & Italie & $\mathrm{g} \mathrm{W}$ & 437 & 1362 & 85 & 59 & 42 & 7 & 1 & 2 & 0 & 5 & 6536 & 257 & 424 & 249 \\
\hline IB3 & XIII & Italie & $\mathrm{g} \mathrm{W}$ & 8 & 258 & 20 & 195 & 32 & 4 & 6 & 1 & 0 & 85 & 13747 & 295 & 116 & 172 \\
\hline$\overline{\mathrm{IB} 4}$ & XIIII & Italie & $\mathrm{g} \mathrm{W}$ & 5 & 278 & 5 & 48 & 15 & 3 & 6 & 1 & 0 & 40 & 4112 & 170 & 135 & 202 \\
\hline IF 1 & XIV & Italie & $\mathrm{g} \mathrm{W}$ & 2 & 34 & 1 & 10 & 98 & 11 & 1 & 1 & 0 & 19 & 2184 & 86 & 316 & 153 \\
\hline MT1 & XIII-XIV & Maghreb & $\mathrm{g} \mathrm{W}$ & 1 & 102 & 3 & 5 & 22 & 1 & 1 & 1 & 0 & 6 & 2017 & 32 & 57 & 5 \\
\hline MT2 & XIII-XIV & Maghreb & $\mathrm{g} \mathrm{W}$ & 15 & 159 & 6 & 21 & 129 & 1 & 1 & 2 & 0 & 11 & 2222 & 118 & 69 & 35 \\
\hline MT3 & XII-XIV & Maghreb & $\mathrm{g} \mathrm{W}$ & 11 & 951 & 9 & 21 & 134 & 1 & 18 & 0 & 0 & 12 & 2335 & 150 & 42 & 10 \\
\hline MY4 & XIV & France & $\mathrm{g} \mathrm{W}$ & 41 & 135 & 51 & 61 & 223 & 7 & 4 & 9 & 1 & 156 & 33448 & 1000 & 589 & 30 \\
\hline MY4 & XIV & France & $\mathrm{g} \mathrm{W}$ & 4 & 102 & 3 & 3 & 29 & 2 & 0 & 1 & 0 & 3 & 1985 & 89 & 355 & 49 \\
\hline MY5 & XIV & France & $\mathrm{g} \mathrm{W}$ & 9 & 27 & 3 & 8 & 25 & 3 & 0 & 0 & 0 & 4 & 2554 & 70 & 810 & 239 \\
\hline EM1 & XIII-XIV & Espagne & g B & 5724 & 15449 & 587 & 124 & 196 & 10 & 1 & 1 & 1 & 7 & 27546 & 383 & 952 & 349 \\
\hline EM2 & XIII-XIV & Espagne & g B & 2987 & 6650 & 465 & 101 & 141 & 10 & 1 & 1 & 1 & 8 & 17918 & 284 & 1367 & 170 \\
\hline EV1 & XIV & Espagne & $\mathrm{gB}$ & 2164 & 717 & 52 & 37 & 10 & 15 & 90 & 1 & 1 & 5 & 13764 & 99 & 352 & 28 \\
\hline EV2 & XIV & Espagne & g B & 2396 & 744 & 72 & 55 & 18 & 8 & 35 & 0 & 1 & 6 & 14211 & 108 & 550 & 259 \\
\hline EV3 & XIV & Espagne & $\mathrm{cB}$ & 49696 & 21408 & 1651 & 544 & 365 & 34 & 5 & 8 & 2 & 20 & 267389 & 1263 & 9945 & 1277 \\
\hline EV3 & XIV & Espagne & $\mathrm{g} \mathrm{B}$ & 3095 & 3769 & 139 & $\overline{15}$ & 27 & 9 & 4 & 2 & $\bar{l}$ & 7 & 19545 & 145 & 1501 & 47 \\
\hline EV4 & XIV & Espagne & g B & 1306 & 257 & 37 & 23 & 80 & 10 & 20 & 1 & 1 & 6 & 9900 & 132 & 140 & 80 \\
\hline EV6 & XIV & Espagne & c B & 13358 & 10497 & 650 & 1104 & 152 & 9 & 1 & 23 & 1 & 9 & 104341 & 385 & 6162 & 476 \\
\hline$\overline{\mathrm{BB} 1}$ & XIII & Italie & g B & 842 & 2733 & 145 & 58 & 37 & 16 & 1 & 1 & 1 & 19 & 7676 & 697 & 171 & 244 \\
\hline IB2 & XIII & Italie & g B & 1117 & 2729 & 109 & 136 & 38 & 6 & 1 & 0 & 1 & 3 & 8575 & 537 & 351 & 258 \\
\hline IB3 & XIII & Italie & g B & 2038 & 2479 & 271 & 129 & 53 & 3 & 1 & 1 & I & 21 & 12636 & 270 & 243 & 181 \\
\hline B 4 & XIII & Italie & g B & 1169 & 3712 & 184 & 61 & 62 & 8 & 10 & 2 & 0 & 21 & 7536 & 364 & 183 & 337 \\
\hline FFl & XIV & $\begin{array}{l}\text { Italie } \\
\end{array}$ & $\mathrm{gB}$ & 3826 & 1985 & 314 & 115 & 153 & 22 & 2 & 3 & 0 & 18 & 37884 & 272 & 2667 & 485 \\
\hline MT1 & XIII-XIV & Maghreb & g B & 1693 & 5469 & 162 & 31 & 48 & 2 & 1 & 0 & 0 & 5 & 9333 & 952 & 168 & 133 \\
\hline MT2 & XIII-XIV & Maghreb & $\mathrm{g} \mathrm{B}$ & 3184 & 5272 & 474 & 76 & 255 & 6 & 1 & 1 & 0 & 14 & 20677 & 2741 & 107 & 196 \\
\hline MT3 & XIII-XIV & Maghreb & g B & 4152 & 6748 & 387 & 58 & 384 & 4 & 3 & 2 & 0 & 7 & 30915 & 27395 & 148 & 191 \\
\hline MT3 & XII-XIV & Maghreb & $\mathrm{gB}$ & 1642 & 2340 & 205 & 35 & 223 & 3 & 1 & 1 & 0 & 7 & 11843 & 752 & 72 & 64 \\
\hline MY1 & XIV & France & g B & 3326 & 2030 & 157 & 46 & 39 & 1 & 2 & 1 & 0 & 2 & 18731 & 219 & 1198 & 278 \\
\hline MY2 & XIV & France & g B & 3531 & 2255 & 131 & 55 & 183 & 3 & 0 & 1 & 0 & 3 & 20594 & 156 & 2214 & 313 \\
\hline MY4 & XIV & France & c B & 1502 & 567 & 69 & 16 & 51 & 5 & 0 & 0 & 0 & 3 & 15221 & 123 & 293 & $\overline{55}$ \\
\hline MY4 & XIV & France & gB & 1150 & 607 & 19 & 12 & 24 & 4 & 0 & 0 & 0 & 0 & 3917 & 201 & 779 & 66 \\
\hline MY5 & XIV & France & g B & 2531 & 443 & 50 & 26 & 23 & 3 & 0 & 1 & 0 & 3 & 17288 & 117 & 924 & 245 \\
\hline MY5 & XIV & France & $\mathrm{cB}$ & 4603 & 741 & 79 & 54 & 73 & 4 & 0 & 1 & 0 & 18 & 46907 & 199 & 1067 & 230 \\
\hline MY6 & XIV & France & cB & 20114 & 1941 & 436 & 25 & 283 & 4 & 21 & 9 & 0 & 0 & 93110 & 571 & 2344 & 174 \\
\hline MY6 & XIV & France & gB & 6470 & 1898 & 204 & 30 & 130 & 3 & 23 & 2 & 0 & 2 & 49908 & 419 & 1954 & 211 \\
\hline SBop & XIV & France & $B$ & 2100 & 4000 & 103 & 104 & 58 & nd & nd & nd & nd & nd & 11200 & 5000 & 550 & 707 \\
\hline SBfil & XIV & France & B & 2200 & 5600 & 169 & 135 & 53 & nd & nd & nd & nd & nd & 14000 & 14000 & 75 & 90 \\
\hline CadH2B & XIV & France & B & 1400 & 1360 & 37 & 84 & 23 & nd & nd & nd & nd & nd & 14200 & 7000 & 4000 & 47 \\
\hline EV4 & XIV & Espagne & $\mathrm{gM}$ & 120 & 79 & 4 & 14 & 58 & 9 & 20 & 0 & 1 & 7 & 3939 & 113 & 161 & 66 \\
\hline IB2 & XIII & Italie & $\mathrm{gM}$ & 303 & 766 & 37 & 158 & 29 & 3 & 9 & 10 & 1 & 0 & 2218 & 30753 & 224 & 298 \\
\hline IB3 & XIIII & Italie & $\mathrm{g} \mathrm{M}$ & 70 & 762 & 62 & 304 & 52 & 6 & 14 & 5 & 2 & 58 & 84910 & 39262 & 416 & 523 \\
\hline IB4 & XIII & Italie & gM & 3 & 140 & 8 & 56 & 12 & 1 & 3 & 1 & 0 & 19 & 19054 & 4488 & 121 & 114 \\
\hline IF1 & XIV & Italie & $\mathrm{g} M$ & 66 & 70 & 3 & 39 & 116 & 12 & 5 & 2 & 0 & 21 & 3089 & 13162 & 453 & 162 \\
\hline MT2 & XIII-XIV & Maghreb & $\mathrm{cm}$ & 85 & 235 & 22 & 737 & 183 & 2 & 3 & 10 & 5 & 9 & 918 & 62739 & 238 & 202 \\
\hline MY4 & XIV & France & $\mathrm{gM}$ & 707 & 3651 & 3 & 205 & 74 & 2 & 2 & 27 & 4 & 368 & 341819 & 85913 & 1008 & 25 \\
\hline MY3 & XIV & France & $\mathrm{gV}$ & 4 & 586 & 5 & 28 & 291 & 29 & 0 & 1 & 0 & 0 & 1542 & 47 & 19393 & 1722 \\
\hline
\end{tabular}

$0=$ non détecté; nd=non dosé; Type et couleur : $\mathrm{g}=$ glaçure, $\mathrm{c}=$ =nene d'application d'une couleur, $\mathrm{W}=\mathrm{blanc}, \mathrm{B}=\mathrm{bleu}, \mathrm{M}=$ marron, $\mathrm{J}=$ jaune, $\mathrm{V}=\mathrm{vert}$.

Tableau 3 : Composition en èlèments traces des glaçures appartenant au groupe 2 défini pour les verres (ppm). 


\begin{tabular}{|c|c|c|c|c|c|c|c|c|c|c|c|c|c|c|c|c|c|}
\hline Réfërence & Epoque & Origine & $\begin{array}{c}\text { Type et } \\
\text { couleur }\end{array}$ & Co & Zn & In & Ni & As & Bi & W & Mo & U & Cr & Fe & Mn & Cu & Sb \\
\hline EB2 & XV & Espagne & g W & 35 & 67 & 2 & 16 & 339 & 5 & 10 & 1 & 2 & 11 & 6177 & 220 & 170 & 250 \\
\hline EV7 & XIV-XV & Espagne & $\mathrm{g} \mathrm{W}$ & 46 & 652 & 1 & 46 & 42 & 83 & 1 & 1 & 1 & 6 & 3541 & 71 & 226 & 457 \\
\hline EV8 & XIV-XV & Espagne & $\mathrm{g} \mathrm{W}$ & 26 & 30 & 2 & 15 & 34 & 10 & 1 & 1 & 1 & 5 & 2669 & 117 & 513 & 657 \\
\hline EV9 & XIV-XV & Espagne & $\mathrm{g} \mathrm{W}$ & 41 & 38 & 5 & 7 & 56 & 141 & 0 & 4 & 2 & 14 & 5492 & 131 & 485 & 444 \\
\hline DB1 & XV-XVI & Italie & $\mathrm{c} \mathrm{B}$ & 3657 & 2196 & 5 & 1054 & 148 & 6 & 173 & 34 & 1 & 6 & 14042 & 446 & 998 & 123 \\
\hline DB1 & XV-XVI & Italie & $\mathrm{g} \mathrm{B}$ & 1429 & 438 & 4 & 341 & 58 & 3 & 27 & 13 & 1 & 44 & 27354 & 595 & 415 & 7 \\
\hline EB2 & XV-XVI & Espagne & $\mathrm{g} \mathrm{B}$ & 3194 & 86 & 3 & 993 & 511 & 9 & 11 & 77 & 3 & 8 & 14534 & 1311 & 251 & 293 \\
\hline EV7 & XIV-XV & Espagne & $\mathrm{g} \mathrm{B}$ & 6942 & 648 & 1 & 2304 & 266 & 67 & 2 & 9 & 1 & 5 & 8642 & 2117 & 861 & 462 \\
\hline EV8 & XIV-XV & Espagne & $\mathrm{g} \mathrm{B}$ & 5989 & 76 & 2 & 2448 & 329 & 10 & 1 & 30 & 1 & 5 & 11596 & 151 & 1107 & 634 \\
\hline EV9 & XIV-XV & Espagne & $\mathrm{c} \mathrm{B}$ & 51693 & 874 & 5 & 30827 & 4564 & 438 & 0 & 62 & 3 & 14 & 183062 & 1048 & 831 & 464 \\
\hline EV9 & XIV-XV & Espagne & $\mathrm{g} \mathrm{B}$ & 8064 & 97 & 2 & 1097 & 687 & 192 & 1 & 48 & 1 & 4 & 25534 & 176 & 482 & 357 \\
\hline OR224 & XV & France & $\mathrm{B}$ & 1500 & 250 & $<1$ & 2060 & 21 & $<1$ & nd & 36 & 1 & nd & 8400 & 11200 & 2500 & 2 \\
\hline
\end{tabular}

$0=$ non détecté; nd=non dosé; Type et couleur : $\mathrm{g}=$ glaçure, $\mathrm{c}=$ zone d'application d'une couleur, $\mathrm{W}=$ blanc, $\mathrm{B}=\mathrm{bleu}, \mathrm{M}=$ marron, $\mathrm{J}=\mathrm{jaune}, \mathrm{V}=$ vert.

Tableau 4 : Composition en éléments traces des glaçures appartenant au groupe 3 défini pour les verres (ppm).

espagnols et coexiste avec la technique de peinture sur émail.

Pour rendre compte de ces problèmes d'hétérogénéité, nous donnons pour certaines glaçures (tab. 3, 4, 5 et 6 ) plusieurs résultats correspondant à différents points d'analyse à l'intérieur de ces dernières.

\section{A-LE COBALTDES GLAÇURES}

Nous allons, dans un premier temps confronter les résultats obtenus sur les glaçures avec ceux obtenus lors de notre étude effectuée sur le verre (fig. 4 et 5) puis, dans un deuxième temps, nous montrerons rapidement comment les résultats obtenus lors de cette étude nous permettent de mieux définir les sources de cobalt (fig. 6 et 7 ) et éventuellement la nature du safre ou du smalt utilisé.

\section{a - Les carreaux de Mehun-sur-Yèvre}

Parmi les échantillons de carreaux de Mehun-sur-Yèvre, quatre ont des glaçures monochromes, ce sont MY1, 2, 3 et 6 . Les glaçures des trois premiers sont monocouches tandis que la glaçure de MY6 est formée de deux couches (une première couche foncée recouverte d'une seconde plus claire). Les deux autres carreaux sont polychromes peints en bleu et marron sur blanc pour MY4, bleu sur blanc pour MY5 - et composés de deux couches pour les zones colorées (cf. planche photos). Les glaçures des échantillons 4,5 et 6 présentent une certaine analogie; il semble en effet que l'on ait appliqué d'abord la couleur ou une glaçure plombifère qui a été ensuite recouverte d'une couche d'émail (plombo-stannifère). Une partie de la couleur a alors diffusé vers la couche supérieure.

Les résultats obtenus sur les différentes parties analysées montrent que, si l'ensemble des glaçures rencontrées sont de type plombifère ou plombo-stannifère, les sous-couches (couleurs bleu et marron) sont essentiellement plombifères. Les carreaux monochromes bleus, obtenus certainement par trempage, sont eux, par contre, du type plombo-stannifère. On a donc deux modes différents de préparation des couleurs.

L'analyse des éléments traces (tab. 3) montre que les parties bleues présentent des teneurs en zinc et en indium très supérieures aux parties blanches et marron. Les teneurs en arsenic, nickel et bismuth ne sont pas significativement différentes d'une couleur à l'autre. On est donc, sans aucun doute possible, en présence du même type de colorant bleu que celui utilisé pour les verres bleus de la même époque (groupe 2). A titre de comparaison, nous donnons dans le tableau 3 la composition de trois verres provenant des verreries médiévales de La Seube (commune de Claret, Hérault) dont on situe la principale phase d'activité de 1307 à 1348, et de Cadrix (Var) qui a fonctionné entre 1365 et 1385 . Pour la verrerie de la Seube, il s'agit d'un verre bleu opacifié à l'étain "SBop" et d'un filet bleu translucide "SBfil", pour Cadrix, d'un pied de verre "CadH2b".

\section{b - Les sculptures de Della Robbia}

Les deux échantillons attribués à Della Robbia sont des glaçures monochromes. Le premier échantillon, DB1, de couleur gris-bleu est composé de deux couches, une souscouche incolore recouverte d'une couche homogène gris bleu. Le second échantillon, DB2, d'un bleu plus vif, est constitué d'une seule couche bleue.

Les éléments traces liés au cobalt nous permettent de rattacher la glaçure gris bleu au groupe 3: les teneurs en indium, arsenic et bismuth sont en effet très faibles. $\mathrm{La}$ présence de zinc est peut-être, dans le cas présent, liée à celle du plomb ou à celle du cuivre. On note en effet la présence de cuivre en quantité assez importante; cet élément accompagne systématiquement le cobalt dans les verres bleus de ce groupe.

La deuxième glaçure, d'un bleu plus vif, est totalement différente. Elle semble en effet appartenir au groupe 4. A titre de comparaison, nous donnons dans les tableaux 4 et 5 les valeurs obtenues pour deux verres de ces groupes: Orléans224 (OR224) pour le groupe 3 et Chartres7 (CAP7) pour le groupe 4 . Ces verres sont respectivement une pastille bleue de fond de gobelet et un morceau de vitrail datés des deux derniers tiers du XVème siècle.

Ces résultats confirment l'existence du groupe 3 à l'époque charnière que constitue le XVème siècle d'après les données réunies sur les verres. Ils permettent, par ailleurs, d'affiner la chronologie proposée en faisant apparaitre ces deux groupes de façon certaine dès le dernier quart du XVème siècle alors que l'étude menée sur le verre les situait vers la fin du XVème et même plutôt vers le début du XVIème siècle. 


\begin{tabular}{|c|c|c|c|c|c|c|c|c|c|c|c|c|c|c|c|c|c|}
\hline Référence & Epoque & Origine & \begin{tabular}{|l|} 
Type et \\
couleur
\end{tabular} & Co & $\mathrm{Zn}$ & In & $\mathrm{Ni}$ & As & $\mathrm{Bi}$ & W & Mo & $\mathrm{U}$ & $\mathrm{Cr}_{\mathrm{r}}$ & $\mathrm{Fe}$ & $\mathrm{Mn}$ & $\mathrm{Cu}$ & $\mathrm{Sb}$ \\
\hline EB1 & $X V$ & Espagne & $\mathrm{g} \mathrm{W}$ & 47 & 255 & 1 & 37 & 22 & 46 & 10 & 1 & 4 & 5 & 2702 & 55 & 161 & 630 \\
\hline EB3 & XVII & Espagne & g W & 20 & 32 & 6 & 18 & 123 & 636 & 13 & 1 & 6 & 0 & 1670 & 33 & 935 & 28 \\
\hline EB4 & XVII & Espagne & $\mathrm{g} \mathrm{W}$ & 17 & 12 & 3 & 13 & 167 & 127 & 4 & 1 & 1 & 4 & 2286 & 33 & 170 & 234 \\
\hline EB5 & XV & spagne & $\mathrm{g} \mathrm{W}$ & 20 & 23 & 1 & 23 & 109 & 31 & 1 & 1 & 2 & 5 & 2518 & 79 & 57 & 141 \\
\hline FL3 & XVI & France & $\mathrm{g} \mathrm{W}$ & 41 & 87 & 1 & 29 & 124 & 126 & 4 & 2 & 1 & 15 & 2231 & 90 & 1127 & 331 \\
\hline FP1 & XVII-XVIII & France & $\mathrm{g} \mathrm{W}$ & 20 & 11 & 2 & 0 & 66 & 304 & 0 & 0 & 0 & 0 & 9187 & 29 & 170 & 123 \\
\hline FP2 & XVII-XVIII & France & $\mathrm{gW}$ & 4 & 8 & 1 & 21 & 94 & 714 & 5 & 1 & 0 & 3 & 2036 & 28 & 275 & 166 \\
\hline FP3 & XVII-XVIII & France & $\mathrm{g} \mathrm{W}$ & 8 & 39 & 4 & 29 & 158 & 163 & 4 & 1 & 1 & 14 & 1364 & 32 & 170 & 230 \\
\hline IF2 & XVI & Italie & $\mathrm{g} \mathrm{W}$ & 4 & 93 & 1 & 18 & 145 & 35 & 2 & 4 & 1 & 22 & 2722 & 318 & 120 & 73 \\
\hline IF3 & XVI & Italie & $\mathrm{g} \mathrm{W}$ & 149 & 47 & 1 & 81 & 891 & 2234 & 5 & 23 & 0 & 9 & 2898 & 128 & 125 & 145 \\
\hline IS1 & $\mathrm{XVI}$ & Italie & $\mathrm{g} \mathrm{W}$ & 601 & 102 & 2 & 657 & 2222 & 2166 & 2 & 20 & 27 & 37 & 2517 & 83 & 109 & 307 \\
\hline IS1 & $\mathrm{XVI}$ & Italie & $\mathrm{g} \mathrm{W}$ & 402 & 74 & 1 & 495 & 1640 & 1493 & 2 & 12 & 19 & 37 & 3588 & 77 & 62 & 192 \\
\hline IS2 & XVI & Italie & $\mathrm{g} \mathrm{W}$ & 2 & 14 & 1 & 13 & 83 & 99 & 2 & 1 & 1 & 48 & 2156 & 84 & 86 & 142 \\
\hline DB2 & XV-XVI & \begin{tabular}{|l|} 
Italie \\
\end{tabular} & $\mathrm{gB}$ & 8333 & 153 & 11 & 2035 & 6340 & 6035 & 103 & 59 & 2 & 4 & 7650 & 157 & 422 & 141 \\
\hline EB1 & $\mathrm{XV}$ & Espagne & $\mathrm{gB}$ & 3706 & 441 & 1 & 900 & 6000 & 11520 & 10 & 16 & 4 & 7 & 4687 & 169 & 216 & 796 \\
\hline EB3 & XVII & Espagne & $\mathrm{cB}$ & 13599 & 2676 & 2 & 3446 & 31245 & 6807 & 18 & 82 & 66 & 6 & 27625 & 482 & 1789 & 302 \\
\hline EB3 & XVII & Espagne & $\mathrm{cB}$ & 35659 & 1824 & 6 & 8470 & 57956 & 18408 & 50 & 208 & 166 & 10 & 59097 & 8328 & 3823 & 431 \\
\hline EB4 & XVII & Espagne & $\mathrm{gB}$ & 3355 & 38 & 2 & 472 & 1323 & 2237 & 5 & 17 & 5 & 4 & 2997 & 146 & 146 & 161 \\
\hline EB4 & XVII & Espagne & $\mathrm{cB}$ & 10782 & 100 & 3 & 2370 & 33278 & 5839 & 20 & 87 & 63 & 3 & 43922 & 12939 & 327 & 292 \\
\hline EB5 & $X V$ & Espagne & $\mathrm{cB}$ & 2120 & 155 & 6 & 744 & 14232 & 1467 & 11 & 22 & 14 & 37 & 31035 & 7034 & 149 & 66 \\
\hline EB5 & XV & Espagne & gB & 2583 & 58 & 1 & 576 & 1242 & 2651 & 4 & 53 & 3 & 5 & 4196 & 5357 & 80 & 231 \\
\hline FLl & $\mathrm{XVI}$ & France & g B & 4655 & 178 & 1 & 2196 & 6617 & 2538 & 5 & 143 & 1 & 25 & 7398 & 253 & 419 & 926 \\
\hline FL2 & XVI & France & g B & 4618 & 854 & 12 & 1178 & 4491 & 5316 & 8 & 26 & 1 & 7 & 5944 & 242 & 188 & 390 \\
\hline FL3 & XVI & France & $\mathrm{gB}$ & 8513 & 302 & 7 & 2534 & 15827 & 28855 & 22 & 106 & 6 & 22 & 27690 & 190 & 873 & 989 \\
\hline FP1 & XVII-XVIII & France & $\mathrm{gB}$ & 1295 & 37 & 2 & 321 & 185 & 624 & 1 & 4 & 2 & 7 & 3182 & 379 & 317 & 164 \\
\hline FP2 & XVII-XVIII & France & $\mathrm{gB}$ & 3806 & 45 & 2 & 1223 & 11430 & 3029 & 9 & 40 & 246 & 3 & 6861 & 112 & 410 & 108 \\
\hline FP3 & XVII-XVIII & France & $\mathrm{gB}$ & 10476 & 266 & 2 & 4162 & 16295 & 2892 & 2 & 30 & 74 & 13 & 14967 & 5596 & 92 & 274 \\
\hline F2 & XVI & Italie & $\mathrm{cB}$ & 5674 & 73 & 3 & 1344 & 4331 & 2438 & 7 & 32 & 20 & 13 & 8318 & 663 & 315 & 978 \\
\hline IF3 & XVI & Italie & $\mathrm{cB}$ & 6765 & 139 & 3 & 1469 & 27499 & 12106 & 21 & 61 & 117 & 11 & 33849 & 19545 & 294 & 1086 \\
\hline IF3 & XVI & Italie & $\mathrm{gB}$ & 5771 & 122 & 3 & 1241 & 8660 & 8534 & 14 & 81 & 23 & 24 & 7469 & \begin{tabular}{|l|}
317 \\
\end{tabular} & 174 & 272 \\
\hline ISl & XVI & Italie & $\mathrm{cB}$ & 3127 & 102 & 1 & 1250 & 10865 & 2462 & 4 & 27 & 197 & 32 & 10192 & 366 & 98 & 308 \\
\hline IS2 & $\mathrm{XVI}$ & Italie & $\mathrm{cB}$ & 5745 & 72 & 1 & 1971 & 3995 & 1537 & 6 & 26 & 22 & 22 & 8246 & 326 & 168 & 179 \\
\hline CAP7 & $\mathrm{XV}$ & France & $\mathrm{B}$ & 1180 & 180 & $<1$ & 450 & 1480 & 1090 & nd & 8 & 2 & nd & 6900 & 9000 & 50 & 5 \\
\hline EB3 & XVII & Espagne & $\mathrm{c} \mathrm{J}$ & 129 & 2975 & 6 & 276 & 669 & 3219 & 15 & 12 & 4 & 11 & 0 & 147 & 636 & 58419 \\
\hline IF2 & XVI & Italie & $\mathrm{c} \mathrm{J}$ & 45 & 28 & 5 & 36 & 401 & 357 & 26 & 6 & 1 & 13 & 40694 & 375 & 178 & 41455 \\
\hline FL3 & XVI & France & $\mathrm{g} \mathrm{V}$ & 77 & 133 & 1 & 46 & 230 & 231 & 3 & 1 & 1 & 13 & 3332 & 94 & \begin{tabular}{|l|}
5581 \\
\end{tabular} & 633 \\
\hline
\end{tabular}

$0=$ non détecté; $\mathrm{nd}=$ non dosé; Type et couleur : $\mathrm{g}=$ glaçure, $\mathrm{c}=$ zone d'application d'une couleur, $\mathrm{W}=$ blanc, $\mathrm{B}=\mathrm{bleu}, \mathrm{M}=\mathrm{marron}, \mathrm{J}=\mathrm{jaune}$, V=vert.

Tableau 5 : Composition en éléments traces des glaçures appartenant au groupe 4 défini pour les verres (ppm).

\section{c-Les céramiques françaises du XVIème au XVIIÌme siècle}

Ces céramiques françaises proviennent principalement de Lyon et de Provence. Ces six échantillons datés des XVIème, XVIlème et XVIİ̀me siècles appartiennent tous au groupe 4.

Ces résultats confirment ceux obtenus sur les verres de la même période. Les profils de concentrations (fig. 1) obtenus à partir de l'échantillon FL3 qui possède un décor polychrome montrent bien que la plus grande partie de l'arsenic, du nickel et du bismuth présente dans la glaçure est apportée par le cobalt. Ces résultats sont confirmés par ceux des tessons polychromes provençaux (tab. 5). Les valeurs très fortes trouvées en cobalt et en bismuth (jusqu'à $3 \%$ pour ce dernier) pour certains points montrent bien l'hétérogénéité du matériau. Ces analyses ont dû être effectuées sur des zones à forte concentration en pigments.

\section{d- Les céramiques italiennes}

Les céramiques italiennes se divisent en trois groupes qui se répartissent de la seconde moitié du XIIIème siècle jusqu'au XVIème siècle.

Les quatre échantillons de Brindisi datés du XIIIème siècle et l'échantillon de Florence daté du XIVème siècle IF 1 - se rattachent au groupe 2, tandis que l'ensemble des autres échantillons, datés du XVlème siècle (Florence et Savone), fait partie du groupe 4.

Parmi les céramiques italiennes, on trouve plusieurs échantillons polychromes, jaune, violet et marron. Les jaunes sont obtenus à partir d'antimoine, tandis que les 
86

\begin{tabular}{|c|c|c|c|c|c|c|c|c|c|c|c|c|c|c|c|c|c|}
\hline Référence & Epoque & Origine & $\begin{array}{l}\text { Type et } \\
\text { couleur }\end{array}$ & Co & $\mathrm{Zn}$ & In & $\mathrm{Ni}$ & As & $\mathrm{Bi}$ & $\mathrm{W}$ & Mo & $\overline{\mathrm{U}}$ & $\mathrm{Cr}$ & $\mathrm{Fe}$ & $\mathrm{Mn}$ & $\mathrm{Cu}$ & $\mathrm{Sb}$ \\
\hline EG1 & XI-XII & Orient & $\mathrm{g} \mathrm{W}$ & 167 & 156 & 1 & 20 & 26 & 0 & 3 & 0 & 1 & 24 & 10919 & 94 & 656 & 3 \\
\hline EG1 & XI-XII & Orient & $\mathrm{g} \mathrm{W}$ & 166 & 331 & 1 & 10 & 10 & 0 & 1 & 0 & 1 & 19 & 10914 & 81 & 594 & 3 \\
\hline OR2 & XIV & Orient & $\mathrm{g} \mathrm{W}$ & 8 & 48 & 1 & 17 & 11 & 0 & 0 & 1 & 0 & 39 & 3420 & 175 & 1433 & 6 \\
\hline $\mathrm{SA3}$ & $\mathrm{XVI}$ & Samarkand & $\mathrm{g} \mathrm{W}$ & 10 & 57 & 0 & 18 & 73 & 2 & 1 & 1 & 1 & 14 & 6269 & 209 & 367 & 1 \\
\hline SA3 & $\overline{X V I}$ & Samarkand & $\mathrm{g} \mathrm{W}$ & 9 & 104 & 0 & 7 & 88 & 2 & 0 & 0 & 1 & 13 & 5264 & 347 & 703 & 1 \\
\hline SM3 & XIII? & Syrie & g W & 6 & 39 & 0 & 27 & 8 & 1 & 0 & 0 & 0 & 29 & 7428 & 312 & 163 & 56 \\
\hline SM4 & XIII? & Syrie & $\mathrm{g} \mathrm{W}$ & 314 & 77 & 1 & 20 & 78 & 0 & 0 & 3 & 0 & 89 & 14142 & 1528 & 529 & 1 \\
\hline OR1 & XII & Orient & g B & 1504 & 46 & 1 & 6 & 2330 & 8 & 28 & 1 & 1 & 4 & 6081 & 339 & 272 & 117 \\
\hline OR1 & XII & Orient & $\mathrm{gB}$ & 856 & 38 & 2 & 18 & 1143 & 9 & 24 & 0 & 1 & 5 & 4116 & 361 & 265 & 138 \\
\hline EG2 & XI-XII & Orient & $g$ B & 1604 & 45 & 1 & 15 & 2725 & 45 & 1 & 1 & 1 & 18 & 14899 & 393 & $\overline{2179}$ & 410 \\
\hline $\mathrm{SA} 2$ & XV & Samarkand & g B & 4400 & 89 & 0 & 10 & 6201 & 1 & 1 & 1 & 1 & 13 & 26425 & 478 & 143 & 2 \\
\hline OR2 & XIV & Orient & $\mathrm{gB}$ & 390 & 80 & 0 & 125 & 7 & 0 & 0 & 0 & 0 & 12 & 3061 & 192 & 691 & 1 \\
\hline OR2 & $\overline{\mathrm{XIV}}$ & Orient & gB & 2861 & 107 & 11 & 1463 & 197 & 0 & 0 & 41 & 0 & 75 & 31335 & 167 & 693 & 14 \\
\hline OR3 & XIV & Orient & c B & 46676 & 415 & 26 & 11678 & 2384 & 3 & 8 & 274 & 1 & 4278 & 67737 & 778 & 377 & 103 \\
\hline OR3 & XIV & Orient & g B & 2862 & 128 & 7 & 676 & 199 & 1 & 0 & 19 & 0 & 19 & 6826 & 299 & 1517 & 8 \\
\hline OR3 & XIV & Orient & $\mathrm{g} \mathrm{B}$ & 821 & 118 & 4 & 571 & 32 & 0 & 0 & 4 & 0 & 35 & 10383 & 216 & 884 & 5 \\
\hline SM2 & XIII? & Syrie & gB & 2315 & 64 & $\overline{0}$ & 29 & 1270 & 1 & 1 & 3 & 1 & 130 & 20458 & 697 & 305 & 2 \\
\hline SM3 & XIII? & Syrie & gB & 2179 & 42 & $\overline{0}$ & 39 & 2513 & 1 & 0 & 1 & 0 & 28 & 17381 & 317 & 221 & 59 \\
\hline SM4 & XIII? & Syrie & $\mathrm{cB}$ & 3008 & 31 & 0 & 37 & 2525 & 1 & 0 & 0 & 0 & 926 & 41133 & 944 & 296 & 6 \\
\hline $\mathrm{SA} 3$ & XVI & Samarkand & g B & 635 & 72 & 1 & 69 & 193 & 2 & 5 & 1 & 1 & 42 & 10776 & 272 & 177 & 2 \\
\hline$\overline{\mathrm{SA} 3}$ & XVI & Samarkand & g B & 3379 & 156 & 0 & 232 & 902 & 3 & 1 & 1 & 1 & 49 & 8098 & 346 & 582 & 1 \\
\hline EGl & XI-XI & Orient & $\mathrm{gB}$ & 1165 & 2291 & 2 & 0 & 71 & 1 & 2 & 2 & 0 & 9 & 22584 & 178 & 3123 & 14 \\
\hline EG1 & XI-XII & Orient & $\mathrm{gB}$ & 1246 & 2504 & 2 & 28 & 68 & 1 & 2 & 1 & 0 & 10 & 22797 & 182 & 3150 & 13 \\
\hline SA1 & $\mathrm{XX}$ ? & Samarkand & g B & 1606 & 125 & 1 & 24 & 23 & 132 & 2 & 1 & 0 & 0 & 1364 & 70 & 154 & 1582 \\
\hline SA4 & $\mathrm{XX}$ ? & Samarkand & g B & 586 & 126 & 0 & 4 & 1 & 9 & 0 & 0 & 0 & 1 & 1254 & 41 & 411 & 18 \\
\hline
\end{tabular}

$0=$ non détecté; Type et couleur : $\mathrm{g}=$ glaçure, $\mathrm{c}=$ zone d'application d'une couleur, $\mathrm{W}=$ =blanc, $\mathrm{B}=\mathrm{bleu}, \mathrm{M}=$ marron, $\mathrm{J}=$ jaune, $\mathrm{V}=\mathrm{vert}$.

Tableau 6 : Composition en éléments traces des glaçures orientales (ppm).

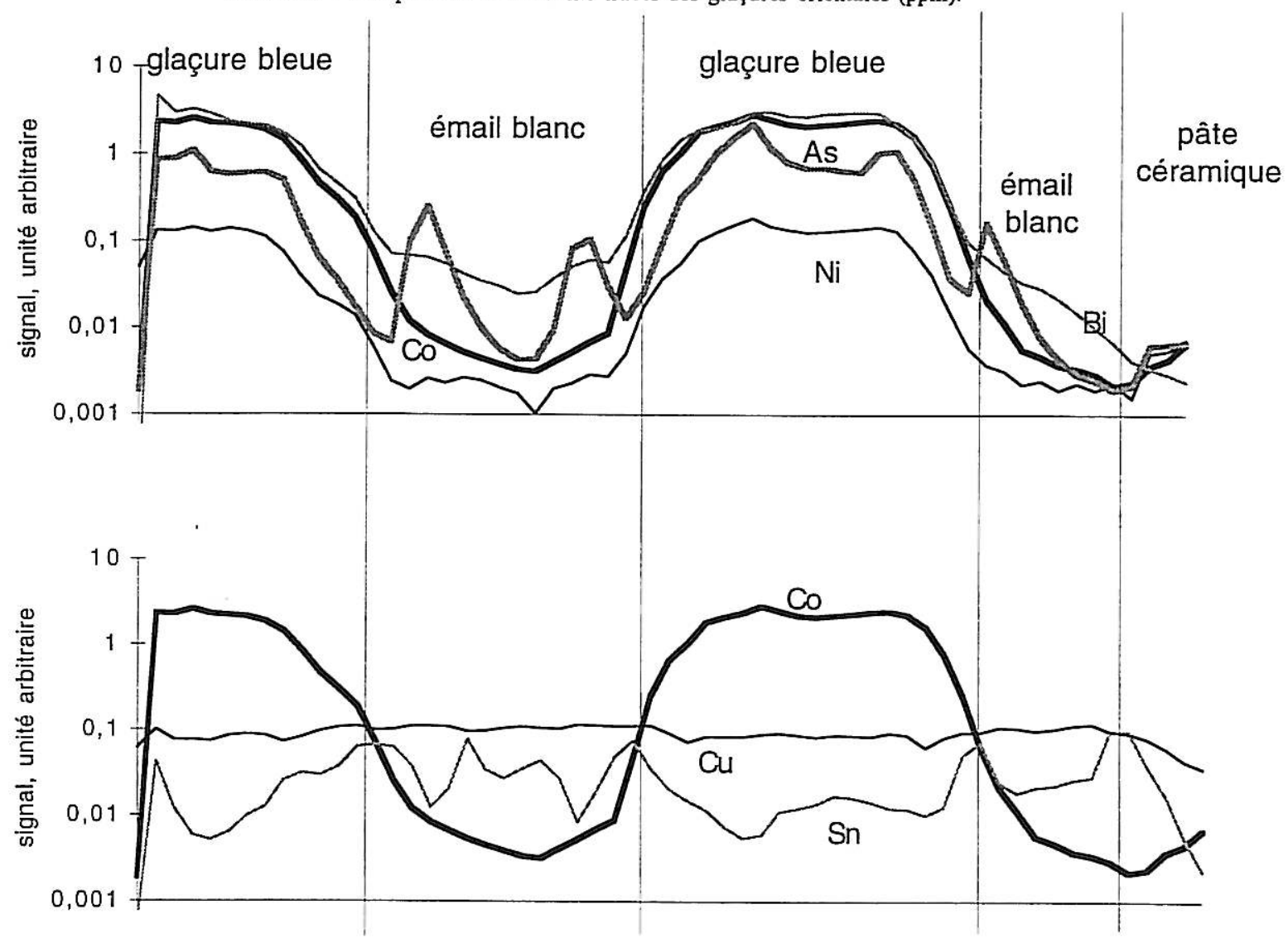

Fig. 1: Profil de concentration pour différents èléments de la glaçure du tesson polychrome FL3. Echelle de déplacement horizontale en points d'analyse, unité arbitraire. 
couleurs brunes et violettes sont dues au manganèse. Les tableaux 3 et 5 donnent les résultats obtenus pour ces glaçures.

Il faut noter ici que l'analyse systématique des couleurs fait ressortir une présence de fer toujours plus élevée dans les décors bleus (tessons FL3 et IFI). Il semble donc que l'oxyde de fer accompagne le cobalt, quel que soit le type de minerai. En effet, IFl est rattaché au groupe 2, tandis que FL3 appartient au groupe 4.

\section{e-Les céramiques espagnoles}

Les céramiques espagnoles s'étagent sur une longue période allant de la fin du XIIIème siècle jusqu'au XVIIème siècle.

Les deux céramiques de Malaga et les six premières céramiques de Valence (les cinq échantillons du "Groupe C" -EVI, 2, 3, 4, 5- et un échantillon "classique", EV6) sont rattachées au groupe 2. La glaçure de EV5 étant très friable, nous disposons uniquement de données qualitatives pour cet échantillon: présence d'indium et absence de nickel, d'arsenic et de bismuth. Ces céramiques sont toutes datées du XIVème siècle; ces résultats confirment, là encore, ceux obtenus à partir des verres.

Les trois autres échantillons de Valence appartiennent au groupe 3. La forte valeur en arsenic trouvée pour EV9 est à rattacher aux fortes valeurs en cobalt et nickel, et pour ce tesson le rapport cobalt/arsenic est supérieur à 10 , il ne peut donc être confondu avec le groupe 4 . Le bismuth est, dans ce cas, certainement amené par plomb, l'arsenic pouvant être associé au plomb ou à l'étain.

Les échantillons de Barcelone se rattachent aussi à ces deux groupes, EB2 appartenant au groupe 3 (comme pour EV9, l'arsenic a probablement une origine différente de celle du cobalt), et les quatre autres échantillons (EB1, 3, 4, 5) au groupe 4.

\section{f - Les céramiques d'Afrique du Nord}

Les trois céramiques tunisiennes datées des XIIIème et XIVème siècles se rattachent au groupe 2. L'analyse des autres teintes confirme la relation entre le cobalt, le zinc et l'indium. Les couleurs brunes sont liées à la présence de manganèse.

\section{g-Les céramiques orientales}

Les résultats obtenus à partir des céramiques d'origine orientale (Proche-Orient, Fustat, Meskéné et Samarkand) sont assez différents de ceux obtenus précédemment. Les minerais de cobalt utilisés pour la coloration de ces céramiques semblent en effet relativement différents de ceux utilisés à partir du XIIIème siècle dans la partie occidentale du Bassin Méditerranéen. L'origine orientale de ces échantillons peut en effet laisser supposer un approvisionnement effectué à partir d'autres sources. Ces échantillons présentant entre eux certaines analogies, nous allons les étudier ensemble.

Parmi ceux-ci, le tesson OR3 est particulièrement intéressant: la préparation de l'échantillon a en effet révélé la présence à l'intérieur de celui-ci d'un grain de couleur bleu (photos 6 et 7). L'analyse de ce grain montre qu'il s'agit d'un verre à très forte concentration en éléments tels que le fer, le cobalt, le nickel et le chrome (tab. 6). Des profils de concentrations effectués à sa surface montrent une forte corrélation cobalt-nickel (fig. 2). L'arsenic, et éventuellement le zinc, pourraient aussi être liés au cobalt. Le cas du chrome et, dans une moindre mesure, celui du fer sont assez différents: en effet, si ceux-ci semblent être systématiquement présents, leurs teneurs varient indépendamment de celle du cobalt.

Il semble que nous soyons ici en présence d'un minerai de cobalt caractérisé par l'association cobalt-nickelarsenic-zinc-fer-chrome, ce dernier élément présentant toutefois de fortes variations de concentration. Signalons aussi que les teneurs mesurées pour cet élément dans l'ensemble des autres glaçures étudiées ci-dessus sont généralement de l'ordre de quelques ppm et excèdent rarement une à deux dizaines de ppm.

L'analyse et les profils de concentrations effectués sur le tesson OR3 (fig. 3) confirment les observations faites sur le grain de couleur. Les résultats obtenus à partir du tesson OR2 semblent aboutir aux mêmes conclusions malgré la très faible teneur en cobalt de cet échantillon.

Ces fortes teneurs en chrome se retrouvent dans les échantillons provenant de Syrie - SM2, SM3 et SM4 - et dans un tesson de Samarkand (SA3). Les résultats obtenus sur ces tessons mettent en effet en évidence une corrélation cobalt-arsenic-chrome. Cependant, à l'inverse de celle observée pour les tessons OR2 et OR3, celle-ci se définit par de très faibles teneurs en nickel et de fortes teneurs en arsenic.

Malgré ces différences, doit-on envisager un lien entre ces échantillons au vu de la seule présence du chrome? Le nombre restreint d'échantillons rend l'interprétation de ces données délicate. Il se pourrait en effet que nous soyions en présence, soit d'un gisement présentant une forte variabilité, soit de plusieurs sources de cobalt (dont l'une

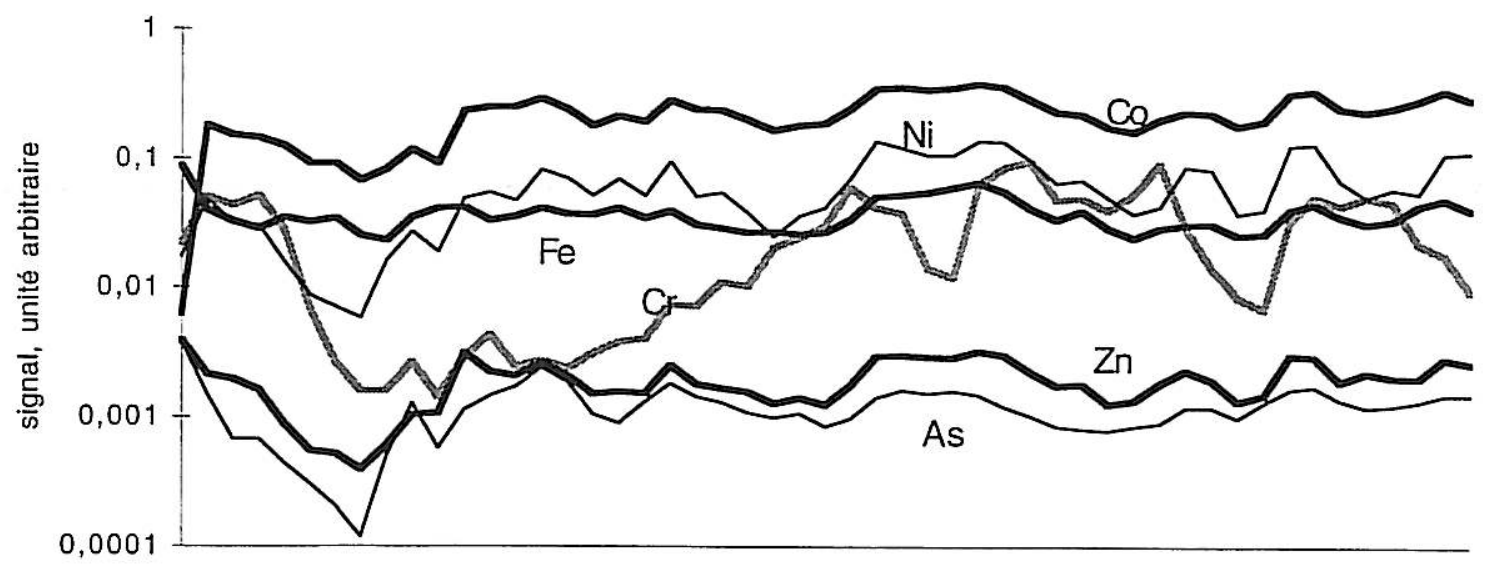

Fig. 2: Distributions des concentrations de différents éléments à la surface du grain bleu du tesson OR3. Echelle de déplacement horizontale en points d'analyse, unité arbitraire. 


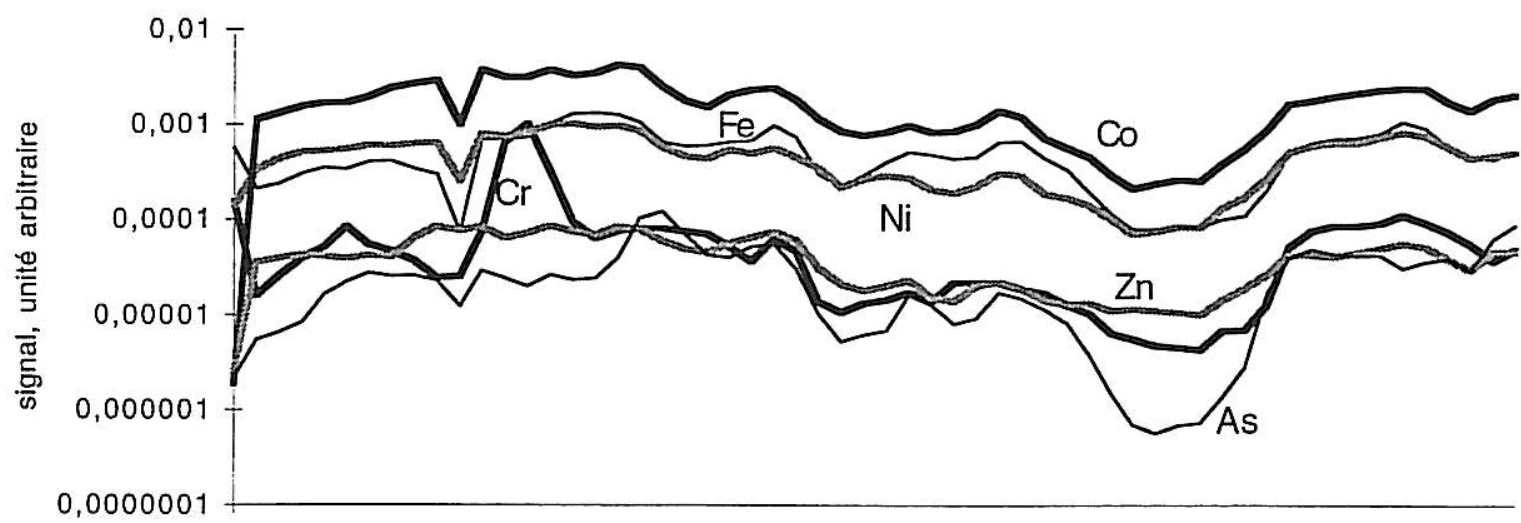

Fig. 3: Distributions des concentrations de différents éléments à la surface de la glaçure du tesson OR3. Echelle de déplacement horizontale en points d'analyse, unité arbitraire.

aussi pourrait présenter une forte variabilité) que seules d'autres analyses pourront nous permettre de caractériser. De plus, il ne faut pas négliger l'hypothèse de mélanges éventuels de minerais de cobalt de différentes provenances.

La question qui se pose maintenant est: d'où provient cette nouvelle source que nous venons d'identifier? Les travaux de Kaczmarczyk sur les faïences égyptiennes (Kaczmarczyk et Hedges, 1983) nous apportent des éléments de réponse. Kaczmarczyk cite en effet la présence de cobalt associé à du chrome, du nickel et du fer en Egypte, à la fois près des anciennes mines d'or de Fawakhir et dans les anciennes exploitations de péridots de l'Ile Saint Jean (Mer Rouge): "Except when it is found in conjunction with cobalt in blue and violet faience of the New Kingdom nickel is as uncommon as chromium. ...... Garnierite and related magnesium silicates, are often found in conjunction with chromic iron in steatite deposits. In Egypt this is true at Fawakhir, near the ancient gold mines, and at the peridot workings on St. John Island."

Un deuxième groupe est constitué par les tessons ORl, EG2 et SA2. Il se caractérise par des teneurs élevées en arsenic et la quasi absence des autres éléments traces habituellement liés au cobalt. On est en présence d'un cobalt relativement pur. Là encore, les travaux de Kaczmarczyk nous apportent de précieux renseignements. Il se pourrait en effet que nous soyions en présence du fameux bleu mahométan ("Mohammedan blue"): d'après Kaczmarczyk et Hedges (Kaczmarczyk et Hedges, 1983), ce cobalt pourrait être originaire d'Iran (mines du village de Qamsar près de Kâshân, mines d'Anarak ou mines de Bayche-Bâgh). Certaines de ces mines peuvent contenir plusieurs variétés de cobalt:

- cobalt rouge, érythrite (arséniate de cobalt, $\left.\mathrm{Co}_{3}\left(\mathrm{AsO}_{4}\right)_{2}, 8 \mathrm{H}_{2} \mathrm{O}\right)$,

- cobalt noir ou terreux, asbolane (manganates hydratés de manganèse et de cobalt de formule générale, $\mathrm{x}(\mathrm{Co}, \mathrm{Mn}) \mathrm{O}$, $\mathrm{MnO}_{2}, \mathrm{yH}_{2} \mathrm{O}$ ),

- cobalt blanc, arséniures et sulpho-arseniures de cobalt (cobaltite, CoAss, skutterudite, $\mathrm{CoAs}_{3-\mathrm{x}}$ ).

A Qamsar, on trouve également des sulfures de fer, de nickel et de cuivre. De telles associations de minerais sont succeptibles d'amener un nombre non négligeable d'impuretés, la composition du pigment obtenu pourrait donc varier de façon importante selon les filons exploités et les traitements effectués au cours des temps (par exemple, des conditions de grillage différentes peuvent influer sur le rapport $\mathrm{Co} / \mathrm{As})$. Il est à noter que ces tessons se répartissent sur une longue période (XIIème-XVème s.) et que de tels changements (filons et modes de traitement) sont plus que probables. Les mines iraniennes semblent être suffisamment riches pour avoir été exploitées pendant plusieurs siècles, certaines l'étaient d'ailleurs encore au début de ce siècle (Kaczmarczyk, 1986).

On pourrait donc interpréter les teneurs rencontrées dans les tessons SM2, SM3, SM4 et SA3 comme étant le résultat d'un mélange de minerais égyptiens ou supposés comme tels et de minerais iraniens.

Un dernier groupe est constitué par le tesson EG1, trouvé en Egypte, qui se singularise par une forte teneur en zinc. De telles teneurs sont assez souvent rencontrées dans les échantillons de verre de la protohistoire (Corse), de l'Antiquité (Gaule) et de l'époque médiévale (Egypte, France) (Soulier, 1997). Sayre cite aussi l'analyse d'un verre provenant de Suse (Crystal scratched vessel from Susa), daté du début de notre ère, et qui présente les mêmes caractéristiques (Sayre, 1964). Ce cobalt semble donc provenir d'un minerai couramment utilisé pendant 1'Antiquité. Il est important de noter ici que les concentrations en manganèse rencontrées dans les échantillons de ce groupe sont relativement faibles. $\mathrm{Ce}$ minerai n'est donc originaire ni des gisements d'alun égyptien (Kaczmarczyk, 1986) ni de petits gisements locaux d'asbolane mais provient certainement d'un gisement important, peut-être situé, là encore, en Iran. On peut éventuellement envisager l'exploitation d'un gisement d'érythrite dans laquelle le zinc se substitue parfois au cobalt.

La nature présumée des tessons SA1 et SA4 (restauration récente?) est confirmée par l'analyse: de par leur composition, ces glaçures se distinguent totalement des autres glaçures provenant de Samarkand. Le cobalt utilisé ici est un cobalt très pur que nous n'étudierons pas plus en détail ici.

La comparaison avec des tessons orientaux nous a permis de relever certaines similitudes entre ce travail et d'autres études. Notre échantillonnage est cependant trop réduit pour formuler des hypothèses concernant les scenarii d'approvisionnement en cobalt de la partie orientale du Bassin Méditerranéen à l'époque médiévale. Seule l'étude systématique des différentes couleurs présentes sur des tessons bien datés et d'origine sûre pourra nous permettre de répondre aux questions posées par ces premiers travaux. Les résultats obtenus pour ces glaçures sont donnés dans le tableau 5. 


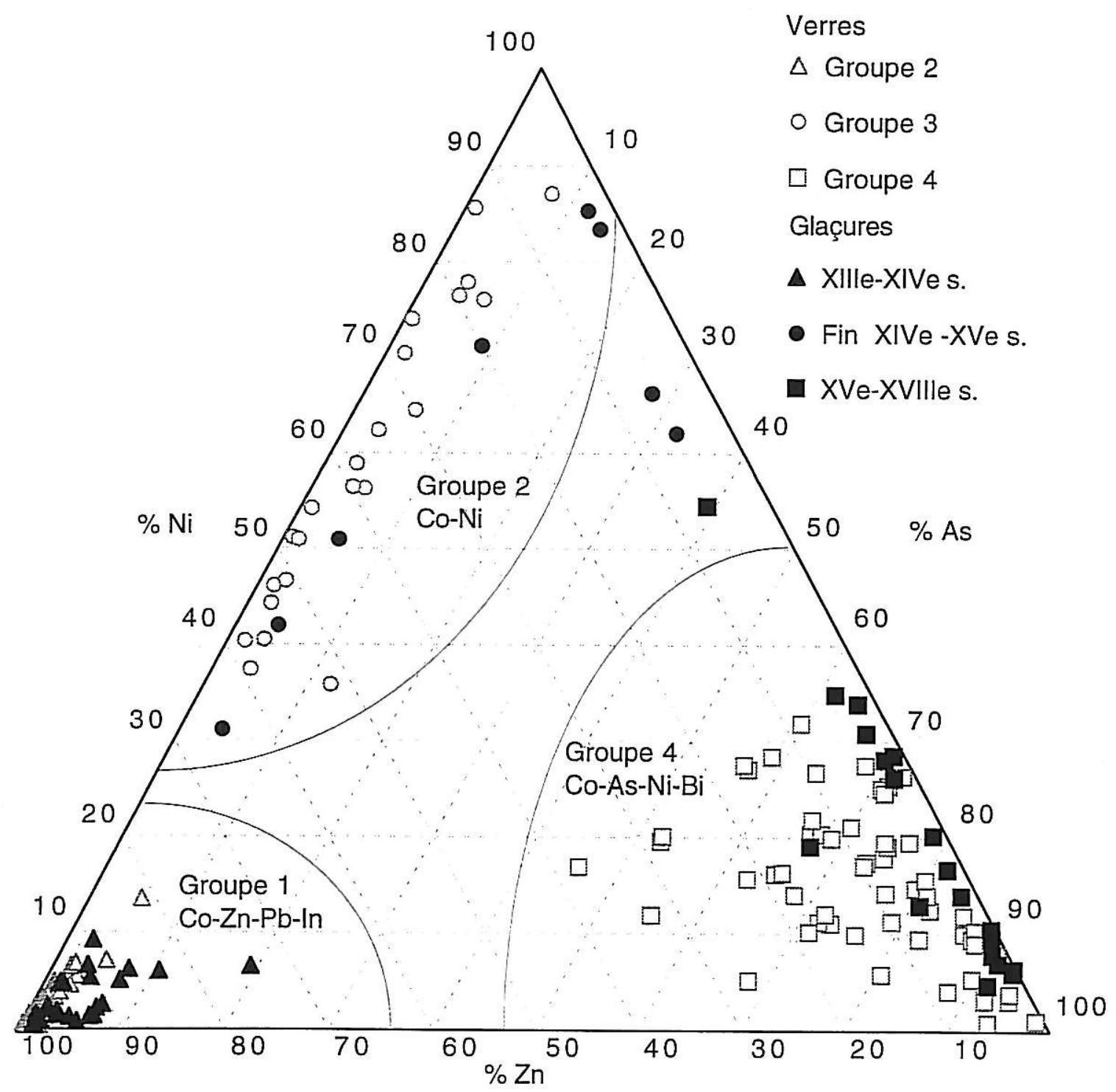

Fig. 4: Diagramme ternaire Ni-As-Zn pour les verres et les céramiques des groupes 2, 3 et 4 (tous les points d'analyse figurent sur le diagramme, une même glaçure peut donc être représentée par plusieurs points).

\section{B - VERS UNE MEILLEURE CONNAISSANCE DES MINERAIS ET DUPIGMENT}

Ces premiers résultats obtenus sur des glaçures de céramique nous ont permis de confirmer certaines corrélations mises en évidence par l'étude de quelques verres par la méthode LA-ICP-MS (Soulier, 1997).

Le diagramme ternaire As-Ni-Zn montre (fig. 4) la bonne concordance entre les données obtenues à partir des verres et des céramiques bleues. Trois points peuvent cependant retenir notre attention sur ce diagramme: il s'agit de deux glaçures attribuées aux groupe 3 (EV9 et EB2) et d'une glaçure attribuée au groupe 4 (FP1) qui se retrouvent en dehors des zones définies pour ces groupes. Si pour EV9 et EB2, l'attribution au groupe 3 ne pose pas de problème, (présence d'arsenic liée à d'autres éléments comme nous l'avons signalé pour les céramiques espagnoles), le cas de FPl est légèrement différent: si les faibles teneurs en éléments traces du groupe 4 (tab. 5) qui caractérisent cet échantillon sont liées à la faible concentration en cobalt de l'échantillon, elles peuvent aussi être interprétées comme le signe d'un début de diversification des approvisionnements en cobalt au XVIIIème siècle. Dans ce cas, l'attribution de FP1 au groupe 4 pourrait être remise en question. Nous ne disposons cependant pas, pour l'instant, de données suffisantes concernant les objets de cette période pour infirmer cette hypothèse.

La présence de molybdène, qui avait été mise en évidence, lors de l'analyse de quelques verres, pour les groupes 3 et 4 est confirmée par cette étude (tab. 3 et 4 et fig. 6). Pour ce dernier groupe, deux nouvelles corrélations sont mises en évidence: une avec le tungstène, l'autre avec l'uranium. La présence systématique de ces éléments semble bien, au moins pour les XVème, XVIème et XVIIème siècles, confirmer l'hypothèse d'un approvisionnement en cobalt à partir d'un seul centre fournisseur situé probablement dans le district minier de Schneeberg. Il est à noter que la présence de tungstène pour certaines glaçures espagnoles des groupes 2 et 3 est probablement liée à celle de l'étain et non à celle du cobalt. En effet, pour ces tessons, les concentrations en tungstène des glaçures bleues et blanches sont quasiment identiques.

Un autre apport majeur de cette étude, est constitué par les découvertes fortuites, d'une part d'un grain de colorant 
(safre?) à l'intérieur du tesson OR3 et, d'autre part de glaçures à forte concentration en pigment (safre + plomb?) pour certains tessons espagnols (EV3 et EV9) et français (MY6). Le terme safre désigne toutefois deux produits différents (Soulier, 1997). Il est indifféremment employé pour décrire:

- un verre riche en cobalt réduit en poudre,

- un mélange broyé de sable et de minerai de cobalt grillé.

Si l'on tient compte de ces deux hypothèses, les zones d'application de couleurs (zones plombiferes) peuvent être interprétées comme étant soit:

- un mélange de safre et d'oxyde de plomb,

- un mélange de safre, d'oxyde de plomb, de sable et de fondant.

Les résultats obtenus sur le grain de couleur du tesson OR3 suggèrent que le safre est plutôt, comme le smalt des siècles ultérieurs, une sorte de verre (ou fritte) très riche en cobalt. Cette hypothèse permet de calculer une composition éventuelle de ce produit (tab. 7) en considérant les glaçures des carreaux comme étant un mélange de safre et d'oxyde de plomb (le calcul est effectué en normalisant à $100 \%$ les éléments que l'on suppose avoir été amenés par le safre). Le même calcul a été effectué pour les carreaux de Barcelone (EB3 et EB4) et Lyon (FL3) appartenant au groupe 4 (analyse de la zone riche en pigment, technique de peinture sur émail). A titre de comparaison, les teneurs moyennes obtenues à partir de deux échantillons de smalt datés du XVIIIème siècle et provenant de Sainte Marie-aux-Mines, ainsi que les analyses d'un grain de smalt trouvé dans des céramiques anglaises du XVIIIème siècle (Freestone), sont aussi données.

Pour calculer la composition du safre du groupe 2 , on a tenu compte de la présence d'un peu de plomb comme l'indiquent les résultats obtenus lors de l'étude effectuée à partir du verre (Gratuze et al., 1992).

Les résultats obtenus pour ces safres d'origines différentes montrent que jusqu'au XVème siècle, le safre semble être un verre très riche en oxyde de fer et de cobalt, ce dernier étant accompagné d'un cortège plus ou moins important d'autres oxydes métalliques dont les teneurs peuvent varier assez fortement à l'intérieur d'un même groupe.

A partir du XVIème siècle, on a un matériau contenant nettement moins d'oxyde de fer. Ceci correspond peutêtre à la mise en place d'une nouvelle méthode de fabrication - méthode de Peter Weidenhammer en 1520? (Lange, 1959) - ou à l'exploitation de minerais plus purs.

Ces calculs ne sont, bien sûr, que des estimations et il se peut que d'autres oxydes (silice, fondant) aient été ajoutés au mélange safre-oxyde de plomb ou que certains éléments proviennent d'une contamination - pâte céramique ou émail - lors du prélèvement effectué par le laser. Là encore, seule la découverte et l'analyse d'autres grains de "safre" comme celui trouvé dans le tesson oriental pourra nous permettre de mieux comprendre la nature de ce matériau.

\section{CONCLUSIONS}

Ces premiers résultats obtenus sur des glaçures de céramiques confirment les hypothèses développées à partir de l'étude réalisée sur le verre: à partir du XIIIème siècle, il existe bien en Europe, et aussi semble-t-il au Maghreb, un réseau de diffusion principal du cobalt à partir de grandes mines exploitées successivement. Si l'exploitation des gisements répertoriés - tels ceux de Freiberg et Schneeberg en Allemagne - est confirmée, il ne faut cependant pas négliger ceux d'Espagne, du Maroc, d'Egypte, d'Iran, et de Chine (Porter, 1997 à paraître) qui sont autant de lieux fournisseurs potentiels, de même que les petits gisements locaux dont on ignore souvent la période d'exploitation.

Dans l'état actuel de la recherche, la prudence reste donc de mise. Les textes médiévaux, tout comme la littérature technique, sont souvent à ce sujet peu prolixes. Les études en cours menées à la fois par Y. Porter et I. Soulier soulignent bien ces difficultés pour appréhender la diffusion du cobalt

\begin{tabular}{|c|c|c|c|c|c|c|c|}
\hline $\begin{array}{l}\text { Période et } \\
\text { provenance } \\
\text { Oxydes et } \\
\text { élément en \% }\end{array}$ & $\begin{array}{l}\text { Orient } \\
\text { OR3 } \\
\text { XIVes. }\end{array}$ & $\begin{array}{l}\text { Co-Zn-In-Pb } \\
\text { EMI } \\
\text { XMle-XIVe s. }\end{array}$ & $\begin{array}{c}\text { Co-Zn-In-Pb } \\
\text { EV3 } \\
\text { XIVes. }\end{array}$ & $\begin{array}{c}\mathrm{Co}-\mathrm{Ni} \\
\text { EV9 } \\
\text { fin XIVe-XVes. }\end{array}$ & $\begin{array}{l}\text { Co-As-Ni-Bi } \\
\text { Barcelone Lyon } \\
\text { XIVe-XVIle s. }\end{array}$ & $\begin{array}{l}\text { Ste Marie- } \\
\text { aux-Mines } \\
\text { XVIIle s. }{ }^{*}\end{array}$ & $\begin{array}{c}\text { Limehouse } \\
\text { Angleterre } \\
\text { XVIIe s. } \\
\neq \neq\end{array}$ \\
\hline $\mathrm{SiO} 2$ & $50-62$ & 50 & 35 & 44 & 70 & 63 & $67,7-69,6$ \\
\hline $\mathrm{K} 2 \mathrm{O}$ & 3 & 4 & 7 & 4 & 9 & 17 & $1,3-1,7$ \\
\hline $\mathrm{Na} 2 \mathrm{O}$ & 5 & 4 & 1 & 2 & 3,5 & 0,3 & $1,3-2,2$ \\
\hline $\mathrm{CaO}$ & 3,5 & 5 & 6 & 12 & $2-4$ & 2 & $2,2-2,8$ \\
\hline $\mathrm{MgO}$ & 1 & 1 & 1 & 1 & 0,4 & 0,3 & $0,6-0,7$ \\
\hline $\mathrm{Al} 2 \mathrm{O}_{3}$ & 3 & 3 & 5 & 7 & 3,5 & 1 & $0,9-1,1$ \\
\hline $\mathrm{Fe}_{2} \mathrm{O}_{3}$ & $12-20$ & 18 & 34 & 23 & $4-6$ & 1,5 & $0,7-0,9$ \\
\hline $\mathrm{CoO}$ & $3-7$ & 3,7 & 7 & 7 & $2-3$ & 1,3 & $3,1-4,4$ \\
\hline $\mathrm{ZnO}$ & - & 5,6 & 3 & 0,1 & 0,2 & 0,01 & $?$ \\
\hline $\mathrm{NiO}$ & $1-2$ & 0,03 & 0,07 & 4,5 & 1 & 2 & $?$ \\
\hline As & 0,25 & 0,03 & 0,05 & 0,6 & $4-6$ & 2 & $5,3-5,7$ \\
\hline $\mathrm{Cr}$ & $0,5-4$ & - & - & - & - & $?$ & $?$ \\
\hline $\mathrm{PbO}$ & - & 5,6 & 3 & - & - & - & 0,8 \\
\hline In & 0,005 & 0,15 & 0,3 & - & - & - & $?$ \\
\hline Mo & 0,03 & - & - & 0,01 & 0,05 & $?$ & $?$ \\
\hline $\mathrm{W}$ & - & - & - & - & 0,01 & $?$ & $?$ \\
\hline $\mathrm{Bi}$ & - & - & - & 0,06 & $1-3$ & $?$ & $?$ \\
\hline $\mathrm{U}$ & - & - & - & - & 0,05 & 0,0005 & $?$ \\
\hline
\end{tabular}

Tableau 7 : Composition moyenne éventuelle du «safre» utilisé par les potiers du XIIIe au XVIIIe siècle. 


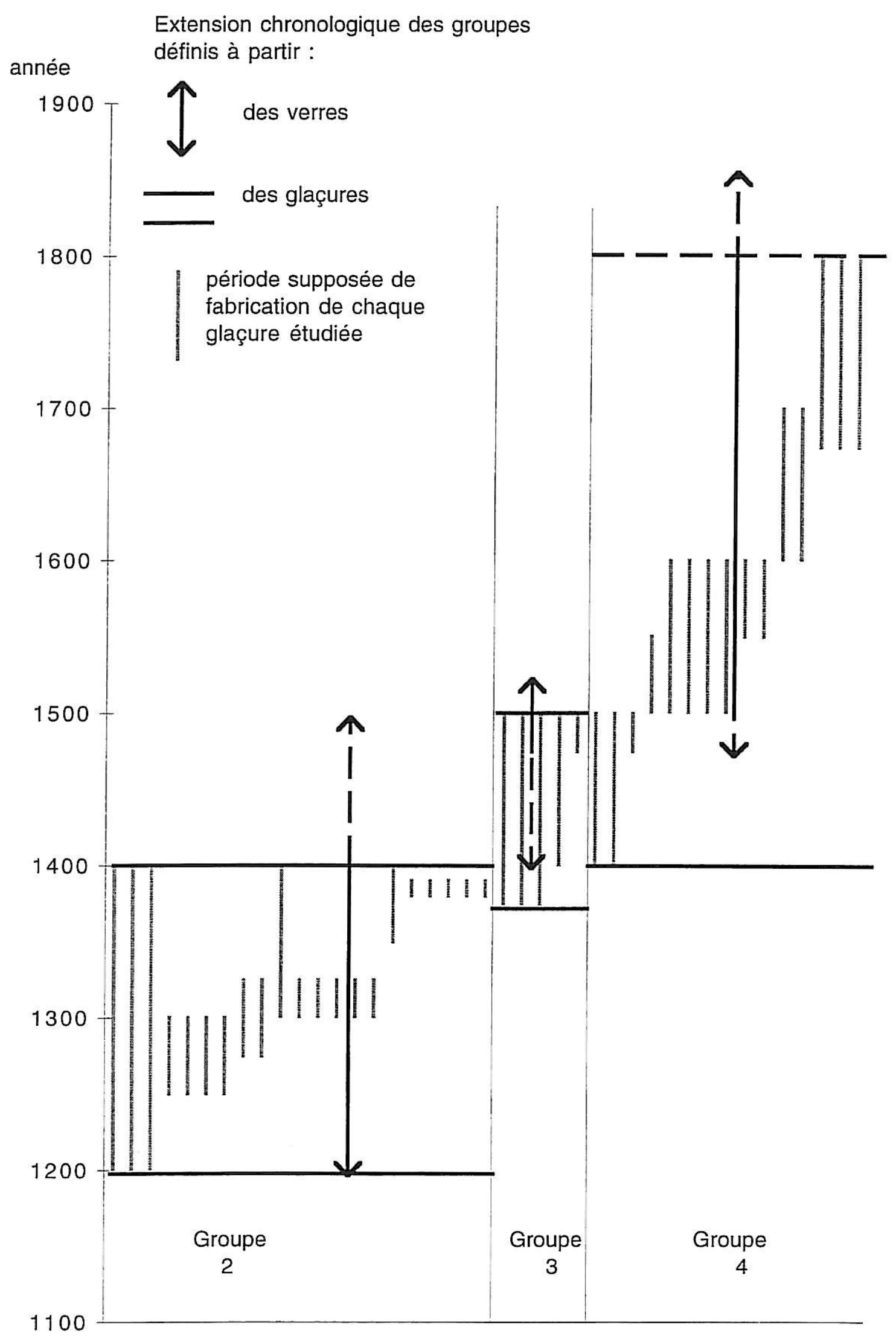

Fig. 5: Comparaison des périodes d'utilisation des diffërents minerais de cobalt définies à partir des verres et des glaçures. 


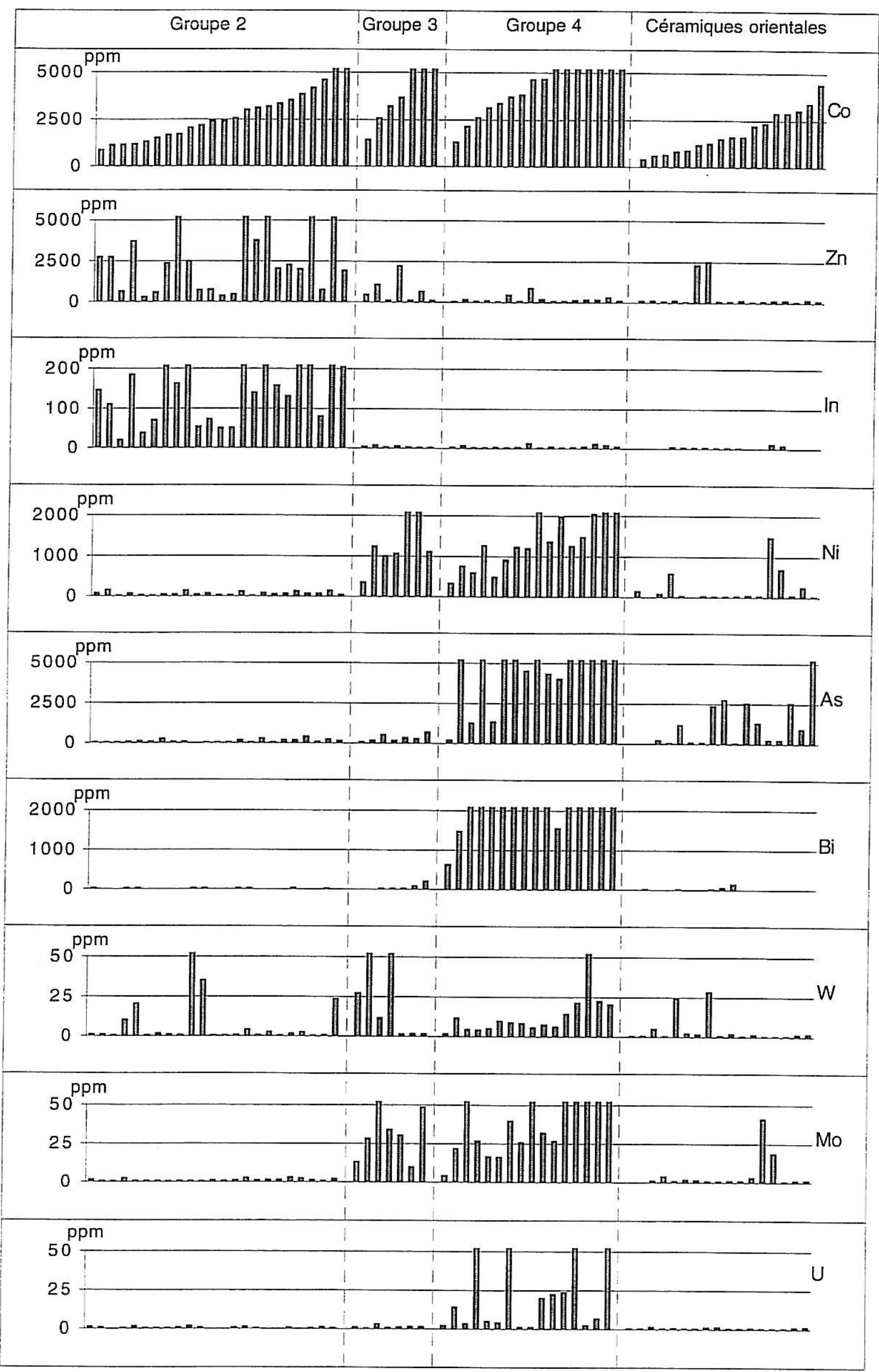

Fig. 6: Histogrammes des teneurs des principaux éléments liés au cobait pour les céramiques étudiẻes en fonction de leur groupe d'appartenance (échelle verticale tronquée pour les fortes valeurs). 


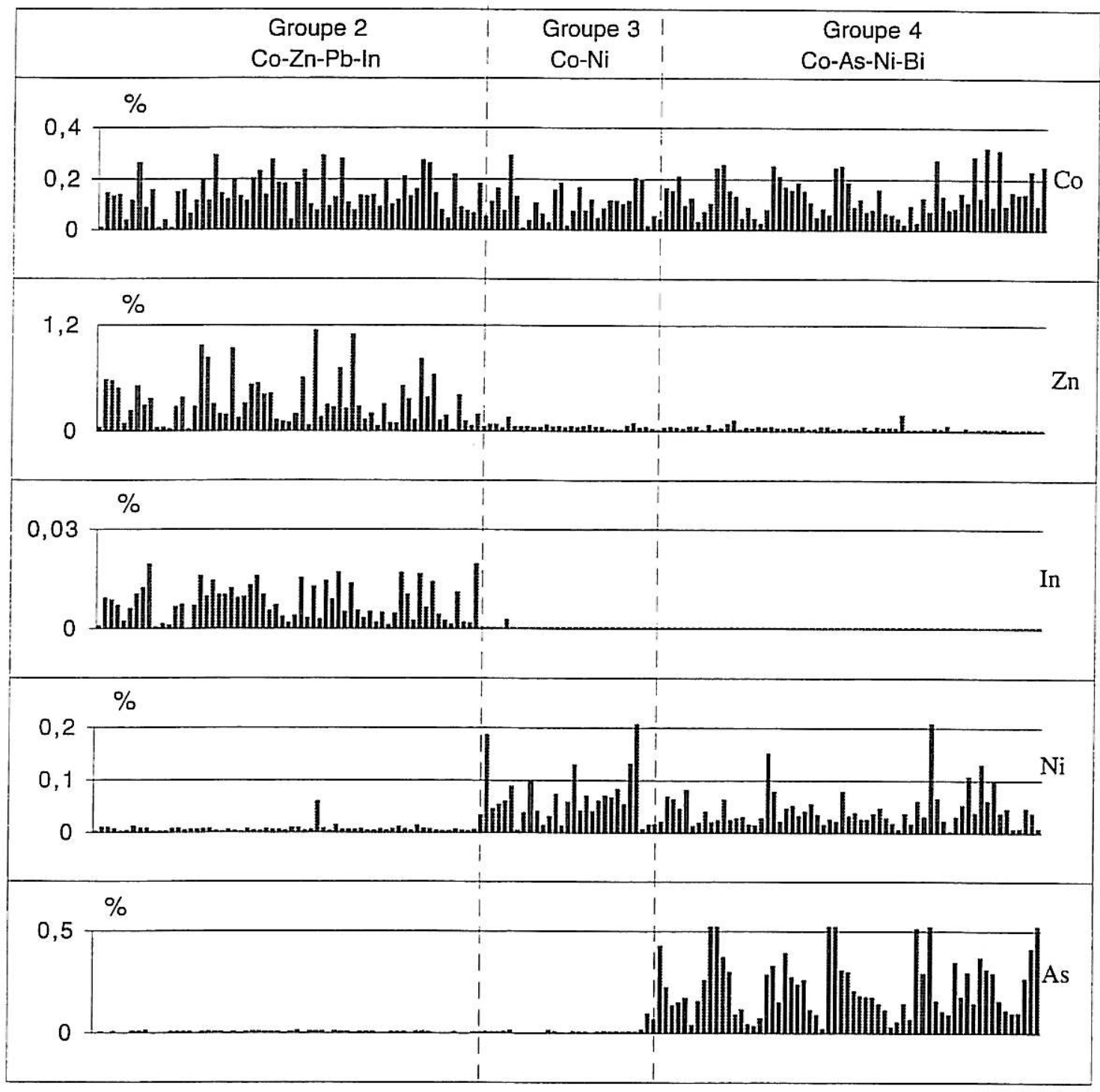

Fig. 7: Histogrammes des teneurs des principaux éléments liẻs au cobalt, pour les verres étudiés lors de nos précedents travaux, en fonction de leur groupe d'appartenance (échelle verticale tronquée pour les fortes valeurs).

qui peut paradoxalement être importé d'Allemagne au XIVème siècle en Iran, tandis que ce même pays producteur exporte son propre minerai vers la Chine!

Ce travail ouvre aussi de nouvelles pistes pour la caractérisation des différents minerais de cobalt utilisés dans la coloration des glaçures et la peinture en bleu des céramiques pour la période s'étalant du Moyen Age à l'époque moderne. Une meilleure définition de certaines de ces sources a été obtenue. Par ailleurs, les analyses effectuées sur certains tessons nous ont permis de mieux comprendre la nature même du pigment utilisé par les potiers et les verriers du XIVème au XVIème siècle.

La chronologie d'utilisation des différentes sources, mise en place lors de nos travaux antérieurs, est affinée par cette étude (fig. 5). Ce travail ouvre aussi de nouvelles perspectives de recherche, concernant d'une part la période antérieure au XIIIème siècle, et d'autre part la partie orientale au sens large du Bassin Méditerranéen. L'extension de cette étude vers les glaçures de céramiques de l'Antiquité est aussi envisagée.

Les résultats obtenus sur les céramiques de Syrie, d'Egypte et d'Ouzbékistan mettent en évidence l'utilisation d'autres gisements de cobalt dont l'un a pu être caractérisé grâce à la découverte d'un grain de pigment à l'intérieur d'une des céramiques étudiées. Une étude systématique des céramiques de ces régions pourrait nous permettre de mieux définir ces sources et éventuellement de mieux comprendre l'approvisionnement en cobalt de l'Occident pour les périodes antérieures au XIIème siècle.

Le problème de l'origine précise des minerais demeure toutefois complexe en l'absence d'un plus grand nombre de références. Seule, la confrontation des données et la multiplication des études menées conjointement par les historiens, les archéologues, les céramologues et les laboratoires scientifiques permettront sans doute de mieux cerner les transmissions de savoir-faire, la circulation et la valeur économique d'une matière présente en Orient comme en Occident.

D'un point de vue analytique, cette étude montre aussi que l'ablation laser couplée à un ICP-MS est une technique très performante pour l'analyse de ce type de matériaux. En effet, la microsonde électronique et la fluorescence $X$, qui étaient jusque là les méthodes les plus utilisées pour ce type d'étude, ne permettent pas le dosage d'autant d'éléments présents à d'aussi faibles concentrations. Ce travail nous laisse aussi entrevoir les possibilités qu'offre cette méthode pour étudier tant l'évolution des recettes et des techniques employées pour la fabrication des glaçures, que les problèmes de provenance. 


\section{REMERCIEMENIS}

Les appareils, VG PlamaQuaủ II et UV MicroProbe, ont été acquis par notre laboratoire gràce à un cofinancement du C.N.R.S. et de la Région Centre.

Les auteurs remercient G. Démians d'Archimbaud, M. Fixot, D. Foy, J.P. Pelletier, J. Thiriot (LAMM), Y. Porter (Université de Provence), M. Moliner (Atelier du Patrimoine, Marseille), A. Hesnard (Centre Camille Jullian, CNRS), J.R. Gaborit (Conservateur Général au Département des Sculptures du Louvre), D. Carru (Service archéologique de Vaucluse), V. Abel, C. Ariaud, A. Horry, F. Parent, (A.F.A.N), P. Bon (Mehun-sur-Yèvre) et B. Goergler pour les échantillons qu'ils ont acceptés de nous confier pour cette étude.

\section{BIBLIOGRAPHIE}

ABEL, V., 1996 - Marseille, futur musée César. Bilan Scientifique, 1995, Aix-en-Provence, DRAC PACA, Service Régional de l'Archéologie, 164-166.

AMOURIC, H., HORRY, A., VAYSSETTES, J.L., 1997 - Le renouvellement des XVe-XVIe siècles en France méditerranéenne: les lieux, les hommes et les produits. In: La céramique médiévale en Méditerranée, Actes du Ve congrès international, Aix-enProvence 1995, à paraître.

BARI, H. et BECKE, A., 1985 - Freiberg, une ville marquée par huit siecles d'exploitation minière, Socièté française de minéralogie et cristallographie, Livret guide, 85

BLET, M., GRATUZE, B. et VALLAURI, L., à paraître - Caractérisation de glaçures archéologiques par LA-ICP-MS, communication proposée au Colloque d'Archéométrie de Rennes en 1997.

BON, P., 1992 - Les premiers "bleus》 de France. Les carreaux de faience au décor peint fabriqués pour le Duc de Berry 1384, édition spéciale du Groupe Historique et Archéologique de Mehunsur-Yèvre, Picard librairie internationale.

CARRU, D., DEMIANS D'ARCHIMBAUD, G., LANDURE, C., PICON, M. et VALLAURI, L., 1995 - Les productions avignonnaises au Moyen Age et à l'époque moderne: état de la question. In: Actes du Se colloque sur la céramique médièvale, Rabat 1991, 292-304.

DE LAUNAY, L., 1913 - Gites minéraux et métalliferes, Paris et Liège (t. II-III).

DEMIANS D'ARCHIMBAUD, G. et LEMOINE, C., 1980 - Les importations valenciennes et andalouses en France méditerranéenne: essai de classification en laboratoire, In: Demians d'Archimbaud, G., Picon, M., dir., La Céramique médiévale en Méditerranée occidentale, Xe-XVe siècles. Valbonne 1978, Paris, C.N.R.S., 359-372.

DEMIANS D'ARCHIMBAUD, G. et PICON, M., 1980 - La céramique médiévale en France méditerranéenne. Recherches archéologique et de laboratoire. In: Demians d'Archimbaud, G., Picon, M., dir., La Céramique médiévale en Méditerranée occidentale, Xe-XVe siècles. Valbonne 1978, Paris, C.N.R.S., 16-42.

DEMIANS D'ARCHIMBAUD, G., THIRIOT, J., VALLAURI, L. et FOY, D., 1980 - Céramiques d'Avignon. Les fouilles de l'hôtel de Brion et leur matériel. Mém. Acad. Vaucluse, 7e série, t. I, Avignon: èd. Aubanel, 195 p., fasc. hors-série.

DEMIANS D'ARCHIMBAUD, G., 1981 - Les fouilles de Rougiers. Contribution à l'archéologie de l'habitat rural médieval en pays mediterranéen. Paris, CNRS, 724 p.

FLXOT, M., GUYON, J., PELLETIER, J.P. et RIVET, L., 1986 Des abords du forum au palais archiépiscopal. Etudes du centre monumental d'Aix-en-Provence. Bull. monum., 144, (III), 159 290.

FOY, D., RICHEZ, F. et VALLAURI, L., 1995 - La céramique en usage dans l'atelier de verrier de Roquefeuille (Pourrières, Var), exemple d'un dépotoir domestique dans la première moitié du XVIIIe siècle, Archèol. Midi médièv. IV, 135-146.
FREESTONE, I.C., - A technical study of Limehouse ware, In: Freestone, I.C., Hutchinson, M., Pontin, L.H., Watney, B.M. et Werner, A., Limehouse Ware Revealed, èd Drakard, D., English Ceramic Circle, 68-77.

GRATUZE, B., BARRANDON, J.N., DULIN, L., et AL ISA, K. 1992 - Ancient glassy matérials analyses: a new bulk non destructive method based on fast neutrons activation analysis with a cyclotron, Nucl. Instr. and Meth., B71, 70-80.

GRATUZE, B., GIOVAGNOLI, A., BARRANDON, J.N., TELOUK, P. et IMBERT, J.L., 1993 - Apport de la méthode ICP-MS couplée à l'ablation laser pour la caractérisation des archéomatériaux, Revue d'Archéométrie, 17, 89-104.

GRATUZE, B., SOULIER, I., BARRANDON, J.N. et FOY, D., 1992 - De l'origine du cobalt dans les verres, Revue d'Archeometrie, 16, 97-108.

HESNARD, A., PASOUALINI, M. et VALLAURI, L., 1993 Tant va la cruche à l'eau. In: Amouric, H., Abel, V., dir., Un goût d'Italie: céramiques et céramistes italiens en Provence du Moyen Age au XXe siècle. Catalogue de l'exposition, Aubagne, ed. Narration, $19-20$

KACZMARCZYK, A. et HEDGES, R.E.M., 1983 - Ancient Egyptian Faience, éd.Warminster, Angleterre.

KACZMARCZYK, A., 1986 - The source of cobalt in ancient Egyptian pigments, Proceedings of the 24th International Archaeometry Symposium 1984, éd J.S. Olin et M.J. Blackman, Washington.

LANGE, A, 1959 - Das sächsische blaufarbenwesen um 1790 in bildern von $A$. F. Winkler.

MOLINER, M., 1990 - Protomajolique et majolique archaīque du XIIIe s. à Marseille. In: Atti XXIIJ convegno internazionale della ceramica Albisola, 201-217.

PARENT, F., 1991 - La Cèramique du bas Moyen Age au Monas tère de Saint-Pierre de l'Almanarre (Hyères) et à l'église SaintPierre d'Hyères (Var). Mémoire de maîtrise dactylographié, 2 vol., Aix-Marseille I, 58 p., ill.

PORTER, Y., 1997 - Origines et diffusion du cobait utilisé en céramique à l'époque médièvale, In: La céramique médiévale en Mèditerranée, Actes du VIe congrès international, Aix-en-Provence, à paraître.

SAYRE, E.V., 1964 - Some Ancient Glass Specimens with Compositions of Particular Archaeological Significance, Brookhaven National Laboratory, New York, BNL, 879, (T-354).

SOULIER, I., 1997 - Les colorants bleus utilisés par les verriers de l'Antiquité à nos jours, Thèse de l'Université de Provence, à paraître.

SOULIER, I., GRATUZE, B. et BARRANDON, J.N., 1996 The origin of cobalt blue pigments in french glass from the Bronze Age to the eighteenth century, Proceedings of the international symposium on archaeometry, Ankara, à paraitre.

THIRIOT, J., 1991 - Céramiques fines islamiques du Midi de la France au Bas Moyen-Age. In: $A$ cerâmica medieval do mediterraneo ocidental. Lisbonne 1987. Mertola, 285-303.

THIRIOT, J., 1995 - Cèramiques fines et orientales. In: De l'Orient à la table du Pape, l'importation des céramiques dans la région d'Avignon aux XIVe-XVIe siècles. Doc. Archéol. Vauclusienne, $5,25-48$.

THIRIOT, J., à paraître - Les ateliers de potiers de Balis: prospection magnétique et fouille.

VALLAURI, L., 1995 - La circulation des céramiques méditerranéennes au Moyen Age: exemples en Provence et Corse. In: Recherches récentes d'archéologie médiévale corse. Actes du séminaire d'archéologie, Ajaccio, avril 1994, Patrimoine d'une île, Patrimoniu isulanu, 1, Ajaccio, DRAC, S.R.A., 69-77.

VALLAURI, L., 1997 - Les céramiques importées. In: Marchesi, H., Thiriot, J., Vallauri, L., dir., Marseille, les ateliers de potiers du XIIIe siècle et le quartier Sainte-Barbe (Ve-XVIIe siècle). Documents d'Archéologie Française, à paraître. 